Aus der Deutschen Heilstätte in Daros. Leit. Arzt: Geheimrat F. Jessen.

\title{
Morphologie und Biologie der Säugetiererythrozyten als Beitrag zur Physiologie des Blutes und zur allgemeinen Zellenlehre.
}

Von

Dr. Hans Kronberger.

Hierzu 2 Textfiguren.

Mit der Bedeutung, welche die roten Blutkörperchen für die Atmung, Gewebsernăhrung sowie für gewisse Immunprozesse haben, ist ihre Aufgabe für den Săugetierorganismus gewiss noch lange nicht erfüllt. Der Erkenntnis von der Wichtigkeit und Mannigfáltigkeit der Erythrozytenfunktionen entspricht das Bedürtnis nach immer neuen Untersuchungsmethoden, die uns immer vollkommeneren Aufschluss über die Morphologie und Biologie der normalen und pathologisch verănderten Erythrozyten geben sollen. Die üblichen Färbemethoden gestatten nur an den unreifen oder pathologisch veränderten roten Blutzellen eine ausgesprochenere Differenzierung, wăhrend die Differenzierungsfahigkeit dieser Methoden für die Normozyten der Saugetiere noch eine sehr beschrănkte ist. Die Studien, welche aus nekrobiotischen Verănderungen der Erythrozyten auf ibren Bau oder ihre Funktionen schliessen wollen, haben bisher so gut wie keine brauchbaren Ergebnisse geliefert. Veine eingehenden Untersuchungen über die Morphologie und besonders über die sogenannten nekrobiotischen Veränderungen der Saugetiererythrozyten ergaben Resultate, welche mir für die Struktur der Erythrozyten von nicht geringerer Bedeutung zu sein scheinen wie für ihre Biologie und ihre Funktionen. Im folgenden sèien in Kürze die Ergebnisse meiner seit acht Jahren in dieser Richtung gehenden Untersuchungen mitgeteilt. Die Bedeutung des zu behandelnden Gegenstandes macht es erforderlich, meine Untersuchungsresultate in Zusammenhang mit den wichtigsten bisherigen Forschungen und Auffassungen $\mathrm{zu}$ bringen. 
I.

Im frischen Nativpräparat zeigt der Säugetiernormozyt die bekannte Form: meist eine kreisrunde Scheibe, deren Durchmesser je nach der Tierart 2, 5-7, $8 \mu$ beträgt; die grösste Randbreite belauft sich etwa auf $2,5 \mu$. Der Peripherie konzentrisch liegt die zentrale Delle. Das Gebilde erscheint im durchfallenden Licht hellgelb und vollkommen homogen. Die Streitfrage, ob die Säugetiererythrozyten in Wahrheit scheiben- oder glockenförmig sind, kann hier bei ihrer Bedeutungslosigkeit für die Funktionen der roten Blutkörperchen unberührt bleiben. Die blosse Betrachtung der Erythrozyten im Nativpräparat und ihre physikalisch-osmotischen Veränderungen in verschiedenen Suspensionsflüssigkeiten machen es wahrscheinlich, dass sie im wesentlichen aus einer derberen, doch semipermeablen Membran und einem zähflüssigen und hochgradig elastischen intramembranösen Inhalt, dem sogenannten Endosoma (W e i d e n r e i c h) bestehen. Die Annahme Ehrlichs und Rolletts, dass dieses Endosoma von einem protoplasmatischen Waben- oder Netzwerk, dem Diskoplasma bezw. dem Stroma als Träger des Hämoglobins, durchzogen sei, erscheint schon im Hinblick auf die Hauptfunktion der Erythrozyten sehr wahrscheinlich.

Schon die komplizierteren morphologischen Verhältnisse der sich entwickelnden und unreifen Erythrozyten (Normoblasten), sowie der Erythrozyten des pathologisch veränderten Blutes (Megaloblasten u. a.) sprechen dafür, dass auch dem anscheinend so homogenen Aussehen der Säugetiernormozyten eine differenziertere Struktur zugrunde liegen muss. Die meisten Erythrozytenfärbungen, namentlich die eigentlichen Strukturmethoden, suchen direkt oder indirekt die Frage za lösen, ob auch in den reifen Săugetiererythrozyten, sei es in morphologischer Beziehung, sei es durch biologische Äquivalente, der Charakter einer vollkommenen Zelle, wie ihn seine kernhaltigen Vorstufen zeigen, gewahrt sei. - Heute gilt nach der Auffassung der meisten Autoren der Säugetiernormozyt für kernlos. Die Entkernung der Normoblasten geht nach Rindfle isch und seiner Schule durch Ausstossung, nach Kölliker und Neumann durch Karyorrhexis vor sich. Diese beiden Theorien haben nur wenig Wahrscheinlichkeit für sich. Dagegen ist die Lehre $P$ appenheims von der Erythroblastenentkernung auf dem Wege der Karyolyse ein- 
leuchtender, schon im Hinblick auf entsprechende Analogien. Dass auch diese Lehre nicht ausnahmslos zutreffend sein kann, werden wir weiter unten sehen. -

Im engsten Zusammenhang mit der Frage nach dem Zellcharakter der Säugetiererythrozyten stehen alle Beobachtungen, welche man durch. Vermittlung bestimmter Färbemethoden an den Erythrozyten bei regenerativen oder degenerativen Prozessen des Blutes machen kann. Es ist natürlich naheliegend, dass man hier z. B. alle nur irgendwie dem homogenen Protoplasma gegenüber differenzierteren Strukturelemente in genetische Beziehung zu einem Kern oder zu Kernresten brachte. Ich weise nur auf die Deutung der Polychromasie und der verschiedenartigen Granulationen und Tüpfelungen hin. Eine befriedigende diesbezügliche Klärung und einheitliche Auffassung ist bis heute noch nicht erzielt worden.

Mit der Deutung und Verwertung der Befunde, die sich unter Umständen durch gewisse differenzierende Färbungen und bestimmte chemisch-physikalische Beeinflussung an den normalen, reifen Säugetiererythrozyten erheben lassen, beschäftigt sich die Nukleoidtheorie. Zuerst hat Brücke im Jahre 1867 durch Borsaureeinwirkung eine Scheidung der Erythrozytensubstanz in eine: aussere Schicht (Oikoid) und eine davon deutlich verschiedene Inhaltsmasse (Zooid) erzielt. Ähnliche Resultate erhielten unter anderen Rollett, Rindfleisch, Wlassow und vor allem Arnold. Den Ergebnissen dieser chemisch-physikalischen Einwirkungen entsprach, nur noch vollkommener, die Differenzierung durch zweckmässige Färbemethoden. Mit wechseIndem Erfolg liess sich an den Säugetiererythrozyten eine mehr homogene, peripherische Zone und der sogenannte „Innenkörper" darstellen. L avdowsky, der zuerst diese Innenkörper wegen ihrer kernähnlichen Struktur (Granula, Retikulum, besondere Färbbarkeit) „Nukleoide" nannte, ist mit Löwit, Arnold und späteren Autoren der Ansicht, dass die Mehrzahl der reifen Erythrozyten des Menschen und der Säugetiere Reste von Kernsubstanzen ent: halten. Auf Grund eingehender eigener Untersuchungen habe auch ich mich der Auffassung genannter Forscher angeschlossen. Doch möchte ich bis jetzt nur diejenigen zentralen Chromatinkörper als Nukleoide, d. h. als echte Kernreste ansprechen, welche sich durch die verschiedenen Methylenblau-Pikrinmethoden 
(Reddingius, Pappenheim, Kronberger) zur Anschauung bringen lassen. Den Beweis dafür, dass es sich bei den von mir beschriebenen zentralen Chromatinkörpern der reifen Säugetiererythrozyten um gewisse Kernsubstanzen handelt, habe ich vor wenigen Jahren chemisch-analytisch gefestigt. Grosso hat meine Befunde nachgeprüft und bestătigt, er schliesst sich meiner Auffassung vollkommen an. Durch vergleichende Kernfärbungen der Froscherythrozyten habe ich zudem damals nachgewiesen, dass Methylenblan allein in einer derartigen Verdünnung, die keine Chromatinfärbung mehr zulăsst, bei zweckmåssiger Pikrinsăurenachfărbung doch noch die schărfste und vorzüglichste Kernfärbung abgeben kann, dass es also wie kein anderer Kernfarbstoff geeignet ist, auch so minimale Kernreste, wie sie in den Săugetiernormozyten vorhanden sein müssen, unter Umstanden noch deutlich darzustellen. Es ist durchaus nicht statthaft, die Nukleoide ausnahmslos als Artefakte zu erklären.

Da die freien Blutplattchen durch die MethylenblauPikrinfărbung so gut wie überhaupt nicht fărbbar sind, müssen sie von den restierenden Kernsubstanzen der reifen Săugetiererythrozyten natura et principio verschieden sein. Sie sind also keinesfalls von den kernbaltigen Innenkörpern der ausgereiften Erythrozyten abzuleiten. Die Frage, ob die Blutplattchen nach Heim, Preisich und Schilling-Torgau ausgestossene Innenkörper von Zellen darstellen, die gewissen Jungformen der Erythrozyten nahestehen, bedarf noch weiterer eingehender Untersuchungen. Trotz aller Theorien ist die Genese der Blutplattchen heute noch nicht mit Sicherheit aufgeklart.

Strukturelemente von besonderer Bedeutung sind nach meinen Untersuchungen bestimmte $\mathrm{Franula}$, die durch verschiedene Methoden an oder in den Saugetiererythrozyten nachweisbar sind. Die Altmannsche Granulalehre hatte bisher nur sehr bedingte Geltung, soweit sie den Anteil der Granula am Strukturbild der Zelle betrifft ( $z$. B. bei der formativen Beteiligung bestimmter Mitechondriengranula an der Bildung gewisser Muskelfibrillenabschnitte). Dagegen hat sie - namentlich seit Arnolds diesbezüglichen Arbeiten - immer mehr an Bedeutung gewonnen für die Physiologie und Biologie der Zelle und ibre spezifischen Funktionen. Ich erinnere hier nur an die vielseitige Bedeutung, welche.den Granulis für die Assimilation, Dissimilation, Synthese 
und spezifische Sekretion zukommt. - Bevor ich die ausserordentliche funktionelle Bedeutung gewisser Erythrozytengranula bespreche, muss ich zunachst kurz das Vorkommen von Granulis, ihre Darstellungsmöglichkeit und besonders ihren Anteil am normalen Strukturbild der Săugetiererythrozyten erörtern. Bekanntlich finden sich, besonders in gewissen Stadien der Entwicklung und Regeneration, in oder an den Erythrozyten oder ihren Vorstufen bisweilen verschiedenartige Granula. So gilt z. B. die Granulation der Substantia granulofilamentosa, nach Cesaris - De mel Substantia metachromaticogranulosa, beute fast allgemein als Merkmal junger Zellformen. Ferrata, Maximow und andere haben das Vorkommen vital-granulärer Erythrozyten im Knochenmark und im Embryonalblut festgestellt. Auf Regeneration deuten Granula, die sich in den Erythrozyten bei den verschiedenen idiopathischen oder posthamorrhagischẹ Anămien darstellen lassen. Die Abstammung dieser Granula, ihre Natur und ihre Funktionen sind zum Teil noch unbekannt, zum Teil lassen sie sich mit Sicherheit von bestimmten Zellanteilen herleiten. Hierzu sind zu zahlen: die Jolly schen Kernkugeln und Kernkörperchen, sowie die granularen, zuweilen $\mathrm{zu}$ charakteristischen Håufchen gruppierten Kernabschnürungen, die $\mathrm{Nageli}$ und Ferrata an den Megaloblasten des embryonalen Menschen- und Kaninchenblutes, sowie des perniziös-anamischen Blutes beschrieben haben. Die meisten dieser Granula gehören nicht zum konstanten Strukturbild der normalen Erythrozyten. Als gewissermassen „intermediare" Produkte bestimmter Entwicklungsstadien regressiver oder progressiver Art können sie nur in zytogenetischer Beziehung Interesse beanspruchen. - An den normalen, reifen Săugetiererythrozyten lassen sich durch die gewöhnlichen Farbemethoden nur selten und dann meist nur vereinzelt Granula nachweisen. So hat Arnold durch Gentianaviolett-Anilinöl-Fărbung oder durch Methylenblau-Eosin bei vielen roten Blutkörperchen eine fein granulierte, lichtere Mitte und einen gewöhnlich homogen gefărbten peripheren Abschnitt nachgewiesen. Die feinen Granula waren teils ungefärbt, teils violett, rötlich oder blau tingiert. Auch mit Hilfe der vielseitigeren Differenzierungsfăhigkeit der Gramschen Farbung suchte Arnold unsere Kenntnis von der normalen Erythrozytenstruktur zu erweitern. Er stellte zunăchst fest, dass sich bei Anwendung dieser Methode einzelne. Erythro- 
zyten oder Anteile von ihnen, gewöhnlich der Zentralteil, langsamer entfărben als andere. Auch ich habe von der Anwendung der Gramschen Methode für die Farbung der Säugetiererythrozyten ausgiebigen Gebrauch gemacht und dadurch die erwahnten A r nold schen Beobachtungen nicht nur bestatigt, sondern durch das deutliche Auftreten von Granulis in manchen Fallen erweitert gefunden. Die beste Darstellung erzielte ich hier durch schwache Verdünnung der angewendeten Anilinfarbstoffe und kurzdauernde Einwirkung dieser Farbstoffe, der Jodjodkaliumlösung und vor allem des absoluten Alkohols. Die in zahlreichen normalen Erythrozyten beobachteten Granula fanden sich meist im zentralen Teil in Form kleinster, blauschwarz gefärbter, kreisrunder Körnchen, die öfter diplokokkenartig nebeneinander lagen und bald unregelmassig, bald in Form konzentrischer Kreise angeordnet waren. Selten waren vereinzelte kleinste Granula in der peripheren Zone der Blutkörperchen zu beobachten.

Über die chemische Natur der grampositiven Erythrozytengranula orientieren uns verschiedene Analogieschlüsse, über die ich weiter unten berichten werde. Sicherlich gehören die meisten der beschriebenen Granula zum Zentralkörper der echten Nukleoide, der zentralen Chromatinkörper. Um mir Aufschluss über die Frage $z u$ verschaffen, welcher Anteil den grampositiven Granulis etwa an der Struktur der Nukleoide zukomme, habe ich verschiedene Versuche gemacht, die Gramfärbung mit der Methylenblau-Pikrinmethode zu kombinieren. Die sukzessive Anwendung beider Farrbungen ergab wenig befriedigende Resultate. Bessere und gleichmässigere Ergebnisse erzielte ich durch folgende Methqde, die in geeigneterer Kombination gleichfalls die Prinzipien der genannten Differentialfarbungen enthălt:

1. Aufgiessen von Methylviolett und Alkohol $70 \%$ āā auf das luftgetrocknete und durch Methylalkohol fixierte Trockenprăparat.

2. Abspülen mit destilliertem Wasser, Aufgiessen der für die Gramfărbung üblichen $\mathrm{L}$ u g o llösung, - vorsichtige Entfärbung durch absoluten Alkohol (Präparat soll hellviolett aussehen!).

3. Aufgiessen von Es bach reagenz, Abspülen mit Wasser, Nachfärbung mit schwacher Eosinlösung, Trocknen des Prăparates nach abermaligem Abspülen mit Wasser. 
Mit der angegebenen Farrbung lassen sich die besten Resultate dann erreichen, wenn man Farbstoffe wie differenzierende Reagentien nur kurz einwirken lasst. Ich unterlasse genaue Angaben, da ich wiederholt beobachtet habe, dass diese Blutfärbungsmethode, ebenso wie so viele andere, je nach der Beschaffenheit des zu untersuchenden Blutes bei durchaus gleicher. Technik in vielen Făllen wesentlich verschiedene Resultate gibt. - Im gut gelungenen Präparate sehen wir bunte Bilder. Alle Erythrozyten zeigen deutlich einen rosa tingierten Randreifen. Immer sind Erythrozyten zu bemerken, die keine Granula zeigen; diese Exemplare sind gewöhnlich homogen graurötlich gefărbt. Sodann treffen wir rote Blutkörperchen, die ebenso dargestellt sind und eine diffuse, feinste, blauschwarze Granulation aufweisen, die Solitărgranula sind hier von einem hellen konzentrischen Hof umgeben. An der Mehrzahl der Erythrozyten finden wir eine scharfe Scheidung. in eine hămoglobinhaltige, homogen graurötlich gefărbte, periphere Zone und einen hellen zentralen Anteil. der

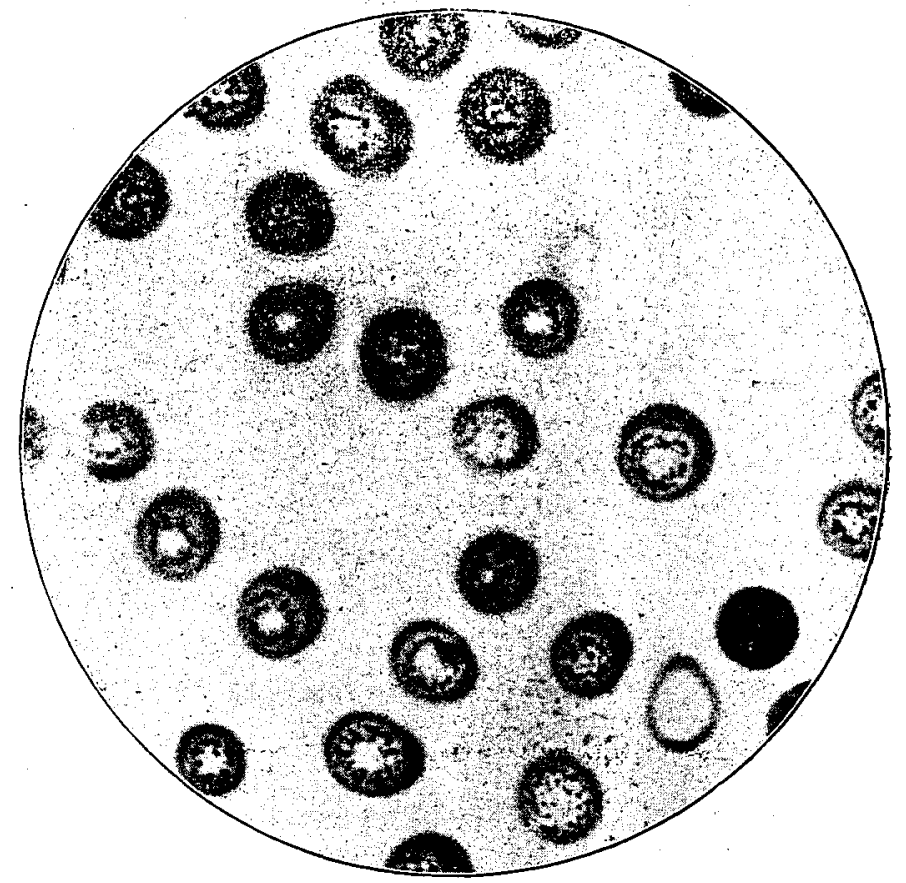

Fig. 1.

Strukturbild normaler Säugetiererythrozyten. Vergr. 1300:1. 
kleinste blauschwarze Granula verschiedener Grösse und Anzahl enthalt, bisweilen in konzentrischen Kreisen angeordnet. - Vielfach ist der zentrale Teil der granulierten Mitte frei von jeglicher Granulation und macht den Eindruck eines glänzenden, bellen Netz- oder Wabenwerkes, in das vielleicht die vermissten Granula eingebettet waren. - In manchen Erythrozyten ist nur die helle kreisrunde Delle ohne jegliche Struktur zu sehen. - Je grösser der granulierte zentrale Anteil, desto heller (hellgrau-rötlich) ist in der Regel die periphere Zone gefärbt. - Der hämoglobinhaltige Anteil der Erythrozyten enthält keine oder nur vereinzelte Granula, wenn das Zentrum in der angegebenen Weise scharf differenziert ist. - Nicht selten liegen die Granula des hămoglobinhaltigen Anteils und des Zentralteiles diplokokkenartig hart nebeneinander. Die durch die kombinierte $\mathrm{Gr}$ a m färbung nachweisbaren Granula gehören mit Sicherheit ebenso wie die durch die einfache G r a mmethode darzustellenden, zum Innenkörper der echten Nukleoide. Freie, d. h. nicht innerhalb der Erythrozyten gelegene Granula habe ich in den gefärbten Trockenpräparaten mit Sicherheit noch nicht feststellen können.

Die verschiedenen Strukturbilder, vor allem die wechselnde und mehr oder weniger ausgesprochene Differenzierung in Granulaform, entsprechen mit grösster Wahrscheinlichkeit verschiedene physiologische oder pathologische intrazelluläre Vorgănge, die durch Methoden wie die einfache oder kombinierte Gr a m färbung fixiert werden. Für diese Annahme sprechen in Fällen schwerer Funktionsschadigungen des Blutes vor allem wesentliche Abweichungen von den gewöhnlichen Strukturbildern, wie ich sie für die Erythrozyten des normalen Blutes beschrieben. habe. Bei schweren infektiösen Intoxikationen, z. B. bei Typhus abdominalis, gelingt es häufig überhaupt nicht, mit Hilfe meiner Kombinationsfärbung Granula in den sonst sehr plastisch dargestellten Erythrozyten nachzuweisen; ausser dem rosa gefürbten Randreifen zeigen sie keine besondere Differenzierung. Interessante Verschiedenheiten gegenüber der Normalstruktur finden wir an den Erythrozyten mitunter bei hochgradigen Anämien. So beobachtete ich z. B. bei einer Sekundäranämie nach profusen Myomblutungen meist Poikilozyten, auffallende Grössenunterschiede der blass eosingefärbten Erythrozyten mit grosser, ungefärbter Delle. Die wenigen Erythrozyten, die normale Grösse aufwiesen, waren 
polychromatisch dunkelgrau-rötlich gefärbt und nur sie allein enthielten spärliche, diffus verstreute, blauschwarze Granula; die Struktur eines Innenkörpers war nirgends auch nur angedeutet.

Die Anschauung, die sich nach meinen Untersuchungen für das normale Strukturbild der Säugetiererythrozyten ergibt, erinnert in mancher Hinsicht an dasjenige, welches Schill ing-Torgau nach seinen Forschungen dem Bau der normalen Erythrozyten zugrunde legt. Dieser Autor unterscheidet am Erythrozyten die $\mathrm{Hb}$-haltige, von einer Lipoidmembran umschlossene Aussenzone und den komplizierter gebauten Zentralteil. Letzterer enthält u. a. den quellungsfähigen, hyalinen, sogenannten "Glaskörper", ferner zwei äusserst kleine, glänzende Körperchen, als „Zentren“ gedeutet, und schliesslich das Blutplattchen. Dass die echten Blutplättchen, wie wir sie frei im Plasma antreffen, keinesfalls von den echten Nukleoiden, den zentralen Chromatinkörpern, abstammen können, habe ich oben dargetan. Ob die Schilling schen Zentren etwa mit Granulis zu identifizieren sind, wie wir sie durch die einfache oder kombinierte Gramfärbung darstellen können, lässt sich allein mit Hilfe rein morphologischer Untersuchungen nicht entscheiden. Die Frage ist aber wahrscheinlich zu bejahen. Ein ăhnliches Körnchen hat schon früher Bremer beobachtet und es zuerst als Paranuklearkörperchen, spăter als Zentrosoma gedeutet und bezeichnet. Türk misst den Erythrozytenzentren grosse Bedeutung bei : „Jeder Untersucher wird z. B. schon oft im Nativpräparate zwei diplokokkenartig mit einander verbundenen glänzenden und tanzenden Körnchen begegnet sein, ohne sie deuten zu können. Mit Schillings Befunden können wir es: Das sind die freigewordenen und isolierten Zentralkörnchen". Auf Grund eigener ausgedehnter experimenteller Untersuchungen schliesse ich mich $\mathrm{T} u ̈ \mathrm{rk}$ an, jedoch nicht nur bezüglich der Bedeutung jener "Zentren“. Im weiteren Verlaufe vorliegender Arbeit werde ich ausführen, dass es Granula sind, die für die Biologie und die wichtigsten Funktionen der Erythrozyten die bedeutendste Rolle spielen. Zuvor jedoch halte ich es für angebracht, zusammenfassend die Struktur der normalen, reifen Saugetiererytbrozyten kurz zu skizzieren, wie man sie nach obigen Untersuchungen und ohne Zuhilfenahme besonderer Hypothesen annehmen muss. 
Der reife, normale Säugetiererythrozyt (Normozyt) stellt ein streng rundes, bikonkaves Scheibchen mit zentraler Delle dar. Er. ist in seiner ganzen Oberflache von einer semipermeablen Lipoidmembran umgeben, an der sich durch besondere Methoden ein schmaler Randreifen zur Anschauung bringen lăsst. Bestimmte chemische und physikalische Einwirkungen sowie zweckmässige Farbemethoden lassen an den Erythrozyten eine homogen sich farbende Aussenzone und einen komplizierter gebauten Innenkörper unterscheiden. Wenn auch Forscher wie Grawitz u. a. bei der Untersuchung im ultravioletten Licht eine besondere Erythrozytenstruktur nicht nachweisen konnten, so weist allein schon die grössere Quellbarkeit der Zentraldelle („Glaskörper ${ }^{4}$ ) darauf hin, dass sie einen reicheren und differenteren Inhalt bergen muss als der periphere Anteil. In jenen Innenkörpern lassen sich durch empfindliche Kernfarrbungen (Methylenblau-Pikrinmethoden) mit Sicherheit Kernreste, echte Nukleoide (zentrale Chromatinkörper) nachweisen, an deren Stelle durch die einfache oder besser durch die kombinierte $\mathrm{G}$ r a m fărbung eine feine granulare Struktur festzustellen ist.

Geistvoll hat bekanntlich Virch ow das Blut ein Gewebe mit flüssiger Interzellularsubstanz genannt. Ich möchte die Erythrozyten als die wichtigsten $Z$ ell en dieses „Gewebes" bezeichnen, denn in den Saugetiernormozyten ist der Zellcharakter vollkommen gewahrt. Die Ursache für die Reduktion und Modifizierung der Normoblastenkerne zu den Nukleoiden der reifen Erythrozyten ist teilweise in verwickelten ontogenetischen Vorgången zu suchen, z. T. kommt in ihnen eine funktionelle Anpassung zum Ausdruck. Die Nukleoide (zentrale Chromatinkörper) sind funktionell und morphologisch den gewöhnlichen Zellkernen aquivalent. Die mehr oder minder grosse Schwierigkeit, die Nukleoide fărberisch darzustellen, ist auf ihre intramembranöse Einbettung zurückzuführen. Dass es heute nicht mebr angangig ist, die reifen Saugetiererythrozyten etwa mit M i not nur als "Blutplastiden", als unvollkommene Zellen, zu bezeichnen, wird sich aus meinen folgenden Erörterungen noch klarer ergeben.

II.

Der wichtigste Teil meiner Arbeit befasst sich mit der Biologie, d. h. mit den Lebenserscheinungen der Ery- 
throzyten. Wie in anderen Zweigen der biologischen Wissenschaft, können auch in der experimentellen Hamatologie die physiologischen Ergebnisse nur dann richtig gedeutet und bewertet werden, wenn man sie kontrollierend und erganzend in entsprechende Beziehung zu den morphologischen Befunden setzt.

Die Struktur der Erythrozyten ist stets der Ausdruck einer Anpassung an innere oder aussere Einwirkungen. Die inneren Einwirkungen treffen die Lebenserscheinungen der Erythrozyten als Zellen am unmittelbarsten. Sie beeinflussen die Struktur besonders der unreifen roten Blutkörperchen in den verschiedenen Zustanden der Entwicklung. An den reifen Erythrozyten machen sich innere Einwirkungen in den verschiedensten Phasen ihres komplizierten Stoffwechsels geltend. Man denke im einen Fall nur an die verschiedenen Modifizierungen, die z. B. die Megaloblastenkerne bis zu ihrer Reduktion zu den echten Nukleoiden der ausgereiften Normozyten erfahren und erinnere sich im anderen Falle an das im wesentlichen gleiche, doch zytologisch individuell differente Strukturbild, wie ich es oben für die normalen Saugetiererythrozyten beschrieben habe! Man vergegenwărtige sich ferner die wesentlichen Abweichungen vom normalen strukturellen Habitus in Fallen, wo schwerere funktionelle Beeintrăchtigungen des Blutes vorliegen (hochgradige Anamien - schwere Intoxikationen). $\mathrm{Zu}$ den intrazellulăren Einwirkungen, welche formatir die Erythrozytenstruktur beeinflussen, gehört in gewissem Sinne auch der verschiedene Hamoglobingehalt, der sich bekanntlich schon in einer verschiedengradigen Affinitut saurer Farbstoffe zur Erythrozytenmembran und damit in ihrer starkeren oder geringeren Farbbarkeit bemerkbar macht. In diesem Zusammenhang darf vielleicht auch die Polychromatophilie und die Möglichkeit, die Substantia metachromatico-granulosa darzustellen, erwahnt werden. Auf eine physiologische Anpassung an mehr aussere; d. h. extrazellulăre Einwirkungen (Osmose, Diffusion!) möchte ich die Ausbildung der Lipoidmembran beziehen, die den Vorstufen der Säugetiererythrozyten fehlt, die wir aber für die reifen und normalen Formen unbedingt annehmen müssen.

Die Lebenserscheinungen jeder Zelle, auch dieder Erythrozyten, sind an ihre Struktur gebunden. Die bedeutendsten Lebenserscheinungen der reifen, normalen Săugetiererythrozyten machen sich vor allem geltend 
in ihrer eigenen $\operatorname{Ernahr} \mathfrak{r} g$ und in ihrer wichtigsten Funktion, welche im engeren Sinne der eigenen Atmung, im weiteren und wichtigsten Sinne der Atmung des Gesamtorganismus dient. Die Ernăhrung der Erythrozyten setzt ihrerseits ein spezifisches Wahlvermögen zur Aufnahme solcher im Plasma gelöster Nahrungsstoffe voraus, welche das Leben der Zellen gewăhrleisten. Es sind dies hauptsachlich die Stoffe, welche zum Aufbau der Lipoidmembran sowie des bei den verschiedenen Säugetierarten differenten Hämoglobins der roten Blutzellen verwendet werden. Es ist schon a priori anzunehmen, dass die wichtigste Erythrozytenfunktion, die Atmung, einzig and allein von der normal ernährten Zelle durch Vermittlung einer bestimmten und im wesentlichen konstanten Struktur geleistet werden kann. Den Beweis für diese Annahme haben die überaus interessanten Arbeiten O. W a rburgs geliefert. Warburg hat vornehmlich auf chemischem Wege die intrazellulären Atmungsprozesse unter Berücksichtigung der Zellstruktur untersucht und gefunden, dass z. B. auch bei den roten Blutzellen die Atmung an ihre Struktur gebunden ist. Die-Oxydationsfăhigkeit einer Zelle ist um so kräftiger, je unversehrter und vollständiger ihre Struktur befunden wird. Als Struktur der Erythrozyten nimmt Warburg ein Gerüstwerk, das Stroma, an, welches gewisse Lipoid- und Nukleinstoffe enthält. - Die von den Säugetiererythrozyten geäusserten Lebenserscheinungen können nach dem Stand unserer heutigen Kenntnisse nur als Leistungen vollkommener Zellen verstanden werden, sie legitimieren gewissermaßen die Berechtigung, mit der wir auf Grund morphologischer und färbungsanalytischer Untersuchungen die Säugetiererythrozyten als vollkommene Zellen bezeichnet haben. Nach unserer heutigen Erkenntnis können nur strukturell und funktionell vollständige Zellen eine derartig bewundernswerte Autonomie aufweisen, wie die Erythrozyten: Vom Muttergewebe der verschiedenen Blutbildungsstätten losgelöst, tragen sie, wie vielleicht nur noch die Eizelle, um mit Virchow zu sprechen, die "Gesetze ihrer Existenz in sich" und bezeugen sie nicht nur innerhalb, sondern unter gewissen Umständen nach der "Explantation" auch noch langere Zeit ausserhalb des lebenden Organismus.

Diese Betrachtungen führen uns zwanglos auf das Gebiet der interessanten Untersuchungen, welche sich mit den sogenannten 
nekrobiotischen Veranderungen der roten Säugetierblutzellen befassen. Schon lange vor den vorhin zitierten Forschungen Warburgs war man sich der grossen Bedeutung der Säugetiererythrozyten bewusst. Da man ihnen aber den Rang vollkommener Zellen nicht einzuräumen wagte, konnte man ihre wichtigen Funktionen nicht in Einklang bringen mit der Sonderstellung, die sie als kernlose Gebilde, als unvollkommene Zellen, einnahmen. Die einfachen morphologischen Untersuchungen mit Hilfe der gebräuchlichen Färbemethoden lieferten nur wenig befriedigende Ergebnisse. Deshalb versuchte man durch das Studium der nekrobiotischen Verănderungen der Erythrozyten über ihre Struktur und insbesondere auch übér ihre vitalen Verhăltnisse genauere Aufschlüsse zu erhalten. Hier sind zu nennen die Untersuchungen Arnold s über die verschiedenartigen Formund Strukturveränderungen, welche die roten Blutkörperchen durch gewisse chemische Agentien (10\% ige Jodkaliumlösung, schwachprozentige Osmiumsăurelösung usw.), sowie bei ihrer Konservierung im hängenden Tropfen, in Glaskapillaren oder in Hollundermark erfahren. Als wesentlichste Resultate der Beobachtungen Arnolds und seiner Schüler (Schultze ú. a.) ergaben sich Abschnürungen der Erythrozyten. Diese Abschnürungen (Schizozyten) waren von verschiedener Form und Grösse, oft den Mikrozyten ăhnlich, waren teils hämoglobinhaltig, teils obne Blutfarbstoff. Die Abschnürungsprodukte waren teils homogen, teils enthielten sie feine Körnungen. Auch Bewegungserscheinungen wurden an verschiedenen Schizozyten wahrgenommen. - Durch Einwirkung höherer Temperaturgrade auf die Blutkörperchen erzielte Schultze ähnliche Gestaltsveränderungen in Form der verschiedenartigsten, meist kugeligen, homogenen Abschnürungen. Erythrozytenabschnürungen der beschriebenen Art lassen sich auch in doppelt unterbundenen Blutgefässen beobachten ( $D$ er e w e $\mathrm{k} \mathrm{o}$ und $\mathrm{Zurhelle}$ ).

Zu umfassenden Aufschlüssen über die Erythrozytenstruktur haben diese Beobachtungen nicht wesentlich beigetragen. Ebenso sind sich die meisten Autoren heute darüber einig, dass der von manchen Forschern (Arnold, Schultze u. a.) angenommene genetische Zusammenhang zwischen gewissen Erythrozytenabschnürungen und den Blutplättchen nicht besteht. - Die Frage, ob es sich bei den geschilderten Abschnürungserscheinungen etwa 
um vitale Äusserungen der Erythrozyten handele, wird heute gleichfalls so gut wie einstimmig verneint. Die beschriebenen nekrobiotischen Vorgănge, die etwa an Lebensăusserungen (Bewegung) erinnern könnten, sind wahrscheinlich zum Teil analog den bekannten $Q \mathrm{u}$ in ckeschen und $\mathrm{B} \ddot{\mathrm{t}} \mathrm{s} \mathrm{chl} \mathrm{l}$ ischen Öltropfenund Ölschaumversuchen zu erklären und sind auf bestimmte Veranderungen zu beziehen, welche Osmose und Oberflächenspannung betreffen. In der Hauptsache handelt es sich also bei den nekrobiotischen Vorgangen, wiesie sich in der Form-und Strukturveränderung der Erythrozytengeltend machen, um Artefakte, die für die Morphologie wie für die Biologie dieser Zellen gleich belanglos sind.

In der Überzeugung, dass die Săugetiererythrozyten autonome Zellindividuen darstellen, sowie in der Hoffnung, auf biologisch-analytischem Wege bessere Einsicht in die Erythrozytenstruktur zu erbalten als mit Hilfe der üblichen Färbemethoden, begann ich im Jahre 1909 meine Untersuchungen über die Nekrobiose der roten Blutzellen. Möglichst ideale Konservierung der Erythrozyten sowie Schaffung von Umstănden, welche die Förderung etwa vorhandener Lebensåusserungen der explantierten Blutzellen gewăhrleisten· und sichtlich potenzieren könnten, war das Prinzip meiner zahlreichen durch Jahre hindurch fortgesetzten Versuche. Diese Versuche, rote Blutzellen analog Bakterien gewissermaßen zu "kultivieren", lieferten je nach Wahl der angewendeten Nährböden verschiedene Resultate. Die Ergebnisse, die ich mit rein flüssigen Năhrmedien erhielt, befriedigten wenig. Die beispielsweise in sterilen verschiedenprozentigen Peptonoder Traubenzuckerlösungen, in physiologischer Kochsalzlösung oder in verschiedenartigen Serumflüssigkeiten suspendierten und verschieden lange einer Temperatur von $37^{\circ} \mathrm{C}$ ausgesetzten Menschen- und Kaninchenerythrozyten zeigten nur Veränderungen, wie sie seit Arnolds Untersuchungen 'bereits zahlreiche Autoren beobachtet haben. Es handelte sich hier lediglich um die oben beschriebenen Artefakte. Dagegen habe ich mit gutem Erfolg zusammengesetzte $\mathrm{Nahrböden}$ verwendet, die den explantierten Erythrozyten gleich dem Blutplasma eiweiss-, kohlehydratund fetthaltige Năhrstoffe sowie Kochsalz zur Verfügung stellen. Am vorteilhaftesten bedient man sich folgenden Nährsubstrates: 


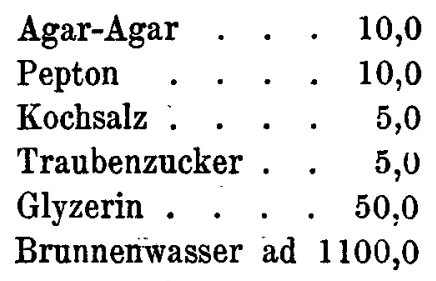

Die Nährbodenmasse wird in weiten Kulturröhrchen nach der Klärung entsprechend den Vorschriften der bakteriologischen Technik an drei aufeinander folgenden Tagen fraktioniert mit grösster Sorgfalt sterilisiert. Die Nährböden sind nur frisch bereitet und nachdem sie auf ihre absolute Sterilităt geprüft wurden, brauchbar und auch nur dann, wenn sie einige Ösen Kondenswasser enthalten. Die Beimpfung der Röhrchen vollzieht sich folgendermaßen: in eine gründlich mit Alkohol und Äther gereinigte Fingerkuppe (beim Kaninchen Ohrvene!) wird mit einer sorgfaltig sterilisierten Lanzette ein Einstich gemacht, der spontan oder auf leichten Druck hin einen genügend grossen Tropfen Blutes austreten lassst. Davon werden mit der vorher ausgeglühten und wieder erkalteten Platinschlinge so rasch als möglich zwei bis drei Ösen zum Teil auf der glatten Agarschicht unterhalb des Kondenswassers verrieben, zum Teil im Kondenswasser selbst aufgeschwemmt. Sodann erhält das Röhrchen einen dicht schliessenden, sterilen Watte-Paraffinverschluss und wird in den auf $37^{\circ \mathrm{C}} \mathrm{C}$ gehaltenen Brutschrank eingestellt.

Die Verănderungen, die wir an den Erythrozyten nach Verlauf verschiedener Zeitintervalle feststellen können, sind zweierlei Art:

1. solche, die denen von Arnold u. a. beschriebenen identisch, die Artefakte sind;

2. solche, die zweifellos auf Lebensäusserungen der explantierten roten Blutzellen zurückzuführen sind.

Rein nekrobiotische Erythrozytenverănderungen, die in der Bildung poikilozyten- und mikrozytenartiger Formen zum Ausdruck kommen, ebenso mehr oder minder ausgedehnter körniger oder netzartiger Zerfall der roten Blutzellen, lassen sich öfter schon wenige Stunden nach Einstellung der Kulturröhrchen in den Thermostaten beobachten. Bei manchen Blutsorten bleiben die Erythrozyten bisweilen acht und mehr Tage ohne jegliche Formveränderung im Kondenswasser konserviert; erst dann treten die eben 
erwähnten Veränderungen auf. Bei meinen Versuchen tritt die Artefaktbildung niemals so ausgesprochen und mannigfaltig auf, wie etwa in den Arnold schen Versuchen. Dies ist darauf zu beziehen, dass eine Suspension der Erythrozyten im hypotonischen Kondenswasser die osmotischen Prozesse der roten Blutzellen rascher eintreten lässt und intensiver gestaltet als eine Aufschwemmung und Konservierung in nahezu isotonischen Suspensionsflüssigkeiten, z. B. im Blutplasma. Es kommt leichtverständlicherweise bei Anwendung eines hypotonischen Suspensionsmediums. eher zu einer Zerstörung der Erythrozyten als zu den bekannten, allmählich sich ausbildenden Abschnürungsprozessen.

Die rein nekrobiotischen Veränderungen der Erythrozyten treten an Bedeutung weit hinter den Erscheinungen zurück, deren Betrachtung ich mich jetzt zuwende. - Wir untersuchen zunächst im Nativpräparat eine Probe der Erythrozytenaufschwemmung einige Tage nach der Einstellung in den Brutschrank. $\mathrm{Zu}$ diesem Zwecke schütteln wir das Kondenswasser des zu prüfenden Röhrchens sanft auf, entnehmen der Kondenswassersuspension einige Ösen und untersuchen sie bei starker Abblendung mit. ölimmersion als hăgenden Tropfen. Die mikroskopische Untersuchung lässt uns kleinste (meist unter Staphylokokkengrösse), ziemlich stark lichtbrechende, grüngelblich gefärbte Körnchen bemerken, die entweder allein oder diplokokken- oder sarżineartig miteinander verbunden angetroffen werden. Die grünlich-gelbe Farbung der Granula ist auf ihren Hämoglobingehalt $\mathrm{zu}$ beziehen. Alle Körnchen sind lebhaft beweglich, die solitären zeigen deutliche Pendelbewegung. Die Zahl dieser Gebilde ist je nach der Sorte der explantierten roten Blutzellen verschieden und wăchst mit dem Alter der „Kulturen“. Unter dem Ultramikroskop zeigen diese Körnchen dieselbe lebhafte Beweglichkeit; sie stellen sich hier als Gebilde mit kreisrundem, goldgelb glänzendem Kern dar, der von einem ihm konzentrischen, schmalen dunklen Ring und einem diesem wiederum konzentrischen, schmalen, goldgelb glänzenden Ring umgeben ist. Ohne weiteres ist nicht zu entscheiden, ob die Beweglichkeit lediglich molekularer oder idiogener Art ist. - Ob die beschriebenen kleinsten Körnchen mit den oben erwähnten beweglichen Körperchen identisch sind, wie wir sie in vielen Nativblutprăparaten sehen, und denen Schilling-Torgau und Türk 
besondere Bedeutung zuschreiben, ist nicht sicher, ich möchte es aber als wahrscheinlich annehmen.

Die geschilderten, im Erythrozytenexplantat auftretenden Körnchen versuchte ich nach verschiedenen Methoden auch farberisch darzustellen. Die Farbung mit den üblichen sauren oder basischen Anilinfarbstoffen (Eosin, Methylenblau, Gentianaviolett u. a.), sowie mit den typischen Kernfarbstoffen (z. B. Hămatoxylin), gelang auf den fixierten Trockenprăparaten so gut wie überhaupt nicht. Die einzige wirklich brauchbare Darstellung im Trockenprăparat lieferte mir bisher die Farbung mit dem gebrăuchlichen Karbolfuchsin, das zur Halfte mit destilliertem oder Brunnenwasser verdünnt wurde. - Die Prăparate zeigen nach dieser Farbung die feinen Körperchen als scharf kreisrunde Granula, die leuchtend hell- oder dunkelrot gefarbt sind. Die grössten Granula sind ungefahr doppelt oder dreifach so gross wie Einzelindividuen einer Kultur von Staphylococcus pyogenes aureus. Die kleinsten Granula sind so klein, dass ihre Grösse noch eben an der Grenze. der optischen Wahrnehmbarkeit steht. Zwischen diesen verhaltnismässig grossen Unterschieden vermitteln zahlreiche Zwischengrössen. Die kleinsten Granula wiegen an Zahl über die grösseren und grössten Formen vor; stellenweise finden sie sich in Kolonien zu vielen Hunderten. - Die Granula aus

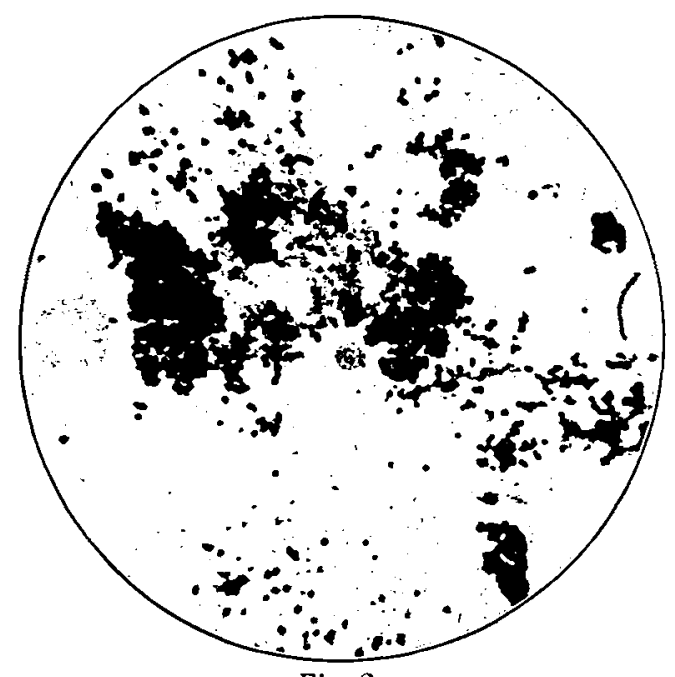

Fig. 2.

Kaltivierte Granula normaler Säugetiererythrozyten. Vergr. 700:1. 
kultivierten Explantaten von Kaninchenerythrozyten sind immer wesentlich grösser als diejenigen ans Explantaten menschlicher Erythrozyten. - Die besten und reichsten Bilder erhalt man bei der Untersuchung alterer (10-14 Tage alter) Kulturen. Sebr vorteilhaft für Darstellung und Studium ist es, die Kondenswasseraufschwemmung alterer Kulturen mit einer entsprechenden Menge Brunnenwasser $\mathrm{zu}$ verdünnen, einmal kräftig zu zentrifugieren und das so gewaschene Zentrifugat zur Herstellung der Trockenpraparate zu verarbeiten und zu fărben. In solchen mit Karbolfuchsin gefăbten Prăparaten finden sich dann gewöhnlich in jedem Gesichtsfeld mehrere grössere Kolonien mit überaus zahlreichen Granulis.

Die Anilinfarbstoffe, die zur fărberischen Darstellung der fixierten Granula unbrauchbar sind, eignen sich doch mehr oder weniger zu ihrer vitalen Fărbung. Besonders gute Bilder gibt die Vitalfărbung mit ziemlich verdünnter, wăsseriger Lösung von Gentianaviolett. Die meisten der einzeln liegenden oder zu grösseren Kolonien vereinigten Granula sind, wie schon erwahnt, frisch aus der Kultur entnommen, lebhaft beweglich. Bald nach Zusatz der Farblösung stellen sie ihre Beweglichkeit ein und fărben sich intensiv blauschwarz. Die Vitalfarbung zeigt deutlich die Entstehung der Granula innerbalb der explantierten Erythrozyten, die bald mehr, bald weniger dieser zarten, kräftig gefărbten Körnchen aufweisen. Abschnürungsformen sehen wir in den vital gefărbten Präparaten (hăngender Tropfen) so gut wie nirgends. - Wir dürfen heute mit Sicherheit annehmen, dass es "Vital"-Färbungen im eigentlichen Sinne nicht gibt, sondern dass bei diesen Methoden lediglich allmählich sich vollziehende pră- oder postmortale Färbungen von Objekten (besonders Granula!) in Frage kommen, die kurz vor der Färbung zweifellos Lebenserscheinungen irgendwelcher Art (vor allem Oxydationserscheinungen!) aufgewiesen haben müssen. Deshalb dürfen wir aus der Wirkung der Vitalfărbung, wie sie in der Verminderung oder Aufhebung der Granulabeweglichkeit zum Ausdruck kommt, den Schluss ziehen, dass es sich bei den oben erwähnten Pendelbewegungen der Granula nicht um Bewegungen nur molekulärer Art handelt, sondern um idiogene Lebensăusserungen von Granulis, die in den roten Blutzellen praformiert sind. 
Über die Herkunft und Entstehung der Granula hat uns bereits die Vitalfärbung Aufschluss gegeben, über ihre Ent wicklung belehren uns die interessanten Bilder, wie wir sie in den von jüngeren kultivierten Erythrozytenexplantaten angefertigten Färbepräparaten sehen. Abschnürungen von Erythrozyten, Mikro- oder Poikilozyten, wie sie A rnold u. a. beschrieben haben, finden sich überaus selten. Gleichwohl treffen wir die Erythrozyten - oft schon wenige Stunden nach der Einstellung in den Brutschrank - mannigfaltig verăndert an. Die roten Blutzellen haben ihre ehemalige kreisrunde Randkontur gewöhnlich verloren, ihre Fläche ist mehr oder minder blassrot gefärbt, in den frühesten Stadien der Verănderung fast homogen, mit Ausnahme feinster, eben sichtbarer Granula, die sich zuerst an den Randpartien der Zellen bemerkbar machen. Im weiteren Verlauf der nekrobiotischen Vorgănge werden die Granula immer deutlicher und verbreiten sich auf der ganzen Fläche, die an Färbbarkeit einbüsst. Schliesslich wird die ganze Erythrozytenfäche nur von diesen Granulis gebildet, während keinerlei Gerüstsubstanz mehr zu beobachten ist. Im frühesten Stadium der Nekrobiose lassen manche Erythrozyten mit fast noch homogener Membran deutlich das Austreten oder die Ausstossung mehr oder weniger zahlreicher Granula feststellen.

Von Kombinationsfärbungen habe ich die von mir angegebene Methylenblau-Pikrinmethode, die Giemsa- und die Gramsche Färbung zur Tinktion der Explantatgranula angewendet. Es gelingt nicht, mit Hilfe der Methylenblan-Pikrinmethode und der Giemsalösung eine brauchbare Fårbung der Granula zu erzielen. Bessere Resultate hatte ich bei Anwendung der Gramfärbung zu verzeichnen. Vorteilhaft ist die Nachfärbung mit Karbolfuchsin, das mit gleichem Volumen Brunnenwasser verdünnt ist. In derart gefärbten Präparaten, die vom Material junger Blutexplantate angefertigt sind, trifft man unter Umstănden in jedem Gesichtsfeld unregelmăssig geränderte Erythrozyten, die mehr oder weniger kraftig rot gefärbt sind und entweder an Stelle der Delle ein grosses, kreisrundes, blauschwarzes Korn führen oder g r a m positive Anteile enthalten, die bezüglich ibrer Form lebhaft an die Kerne der polymorphkernigen Leukozyten erinnern. - Sehr selten - meist nur in älteren Explantaten - beobachtet man Erythrozyten, die zahlreiche kleinste, 
blauschwarze Granula bergen. In grösserer Zahl finden wir rote Blutzellen, die verschieden zahlreiche fuchsingefarbte Granula enthalten. Die extrazellularen Granula sind teils blauschwarz, teils leuchtend rot gefarbt. Unter den ersteren trifft man wiederum solche an, die durchweg grampositiv sind, und solche, welche einen grampositiven kreisrunden Kern und einen gramnegativen Anteil in Form einer schmalen, konzentrischen, fuchsino(azido-) philen Zone aufweisen. Besonders hübsche Bilder liefert die Gramfarbung an gewaschenem und zentrifugiertem Material alterer Kulturen. - Die mit Fuchsinkontrastfarbung kombinierte Grammetbode demonstriert sehr deutlich, dass die grampositiven ebenso wiediefuchsinophilen Explantatgranula innerhalb der roten Blutzellen, zum mindesten and sicherlich aus ihrer Masse entsteben. Die Farbbarkeit nach Gram, welche den freien und intrazellularen Granulis der explantierten Erythrozyten eigen ist, deutet auf die Identitat dieser Granula mit denjenigen, die wir mit Hilfe der einfachen oder kombinierten Gr a m farbung an den fixierten Erythrozyten frisch hergestellter Trockenpraparate darstellen können. - Die azidophilen Granula der Erythrozytenexplantate verdanken ihre Fuchsinophilie wobl sicherlich .einem bestimmten Hamoglobingehalt. Diese Annahme ist schon durch den Hinweis auf die Abstammung unserer Granula von Mutterzellen gerechtfertigt, deren Farbbarkeit durch Fuchsin quantitativ durch einen höheren oder niedrigeren Hămoglobingehalt bedingt ist.

Die von mir beschriebenen Granula aus Erythrozytenexplantaten haben mit gewissen mikroskopischen Befunden Ähnlichkeit. Nur langjăhriges, mühevolles and peinlichst sauberes Arbeiten ermöglichte mir die sichere Entwirrung differentialdiagnostischer Schwierigkeiten. Der Untersucher muss hier ausschliessen können: gewisse Bakterien (Staphylokokken), Abschnürungsprodukte der Erytbrozyten sowie die sogenannten Russelschen Fuchsinkörperchen.

Die Explantatgranula besitzen in gewissen Entwicklungsstadien grosse Ähnlichkeit mit Staphylokokken, die ich - nebenbei bemerkt - auch nicht selten als Mischbakterien im Blute auch fieberfreier Phthisiker kulturell nachweisen konnte. Gegen Staphylokokken sprechen in unserem Falle ausser den betrachtlichen 
Grössenunterschieden der Granula vor allem ihre immer und immer wieder festgestellte Avirulenz im Tierversuch (intravenöse Injektionen !), die Unfăhigkeit der Explantatgranula, multiple makroskopische Kolonien zu bilden, sowie die Unmöglichkeit, sie anf andere flüssigge oder feste Nährböden mit Erfolg überzuimpfen. Ferner fehlt den lebenden Staphylokokken jegliche Beweglichkeit, und schliesslich sind die Färbungsverhältnisse der Staphylokokken vollkommen von denen der Granula verschieden.

Dass es sich bei den in Frage stehenden Granulis keinesfalls um Artefakte im Sinne der oben beschriebenen nekrobiotischen Erythròzytenabschnürungen handeln kann, beweist schon ihre Resistenz gegen verdünnte Essigsäure, welche die artefakten Abschnürungsformen unbedingt zur Auflösung bringt.

Mit den Russelschen Fuchsinkörperchen haben unsere Granula hauptsächlich die besonderen Färbungsverhältnisse (Nichtfărbbarkeit durch die gewöhnlichen Kernfarbstoffe, Azido-Fuchsinophilie) gemeinsam. Gegen die Identität mit den vielbeschriebenen und mannigfach gedeuteten Russélschen Körperchen sprechen vor allem Grössenunterschiede $(0,5-25 \mu)$, wie sie den Explantatgranulis niemals zukommen. Heute gilt es übrigens für sicher, dass genetische Beziehungen zwischen den Ru sse Ischen Fuchsinkörperchen und den Erythrozyten nicht bestehen, dagegen vielleicht genetische Beziehungen zwischen ihnen und Mast- oder Plasmazellen. Die letztere Annahme ist um so berechtigter, als Mastzellengranula ebenso wie die R u s s e l schen Körperchen unter Umständen in gleicher Weise nach Weigert und Russel färbbar sind, und da Sch rid d e phylogenetische Beziehungen zwischen Plasma- und Mastzellen als wahrscheinlich nachgewiesen hat. Meines Wissens sind Russelsche Körperchen bisher noch nie frei im strömenden Blut nachgewiesen worden. Schliesslich habe ich in meinen zahlreichen Blutkulturen noch nie Befunde erheben können, die auch nur irgendwie auf eine Beziehung der Explantatgranula zu den Mast- oder Plasmazellen hindeuteten.

Die von mir beschriebenen kleinsten, kreisrunden Granula, wie sie in der Masse explantierter Säugetiererythrozyten unter der längeren Einwirkung einer Temperatur von $37^{\circ} \mathrm{C}$ entstehen, vermehren sich wahrscheinlich durch Zweiteilung der Einzelindividuen. Diese Fähigkeit, sich zu vermehren, ist bei den verschiedenen Blutsorten eine sehr verschiedene. Dass es sich hier 
tatsăchlich um eine Massenver mehrung der Granula handelt, geht unter anderem daraus hervor, dass die Zahl der einzelnen Granula und Kolonien in der gleichen Kultur von Tag zu Tag steigt. Kame nur die Bildung etwa granulărer Artefakte und ihr Freiwerden aus zerfallenden Erythrozyten in Frage, so ware dieser Vorgang begrenzt und mit dem oft schon nach ganz kurzer Zeit vollstandigen Zerfall der Erythrozyten im. Kondenswasser abgeschlossen. Dementgegen habe ich stets die bisweilen ausserordentlich reiche und stetige Vermehrung der Granula beobachtet, besonders in Explantaten, deren rote Blutzellen rasch, z. B. schon am ersten oder zweiten Tage nach Einstellung in den Brutschrank, vollstandig aufgelöst waren.

In den vorhergehenden Abschnitten haben wir die Bedeutung unserer Granula für die Morphologie, für die Struktur der Săgetiererythrozyten kennen gelernt. Ihre Bedeutung für die Biologie und die Funktionender Erythrozyten lassen sich nur aus ihrer eigenen Biologie ableiten. Von den Lebenserscheinungen der im Erythrozytenexplantat auftretenden Granula haben wir bisher nur ihre Fahigkeit, Năhrstoffe zu assimilieren, ihre Beweglichkeit und ihre Fabigkeit, sich zu vermehren, feststellen können. Tierexperimente müssen in der experimentellen Hamatologie ebenso wie in der experimentellen Bakteriologie die morphologischen und kulturellen Forschungen und Studien ergănzen. Tierversuche haben mir auch überaus wichtige Aufschlüsse über die Biologie unserer Granula und damit auch über die Hauptfunktion der Săgetiererythrozyten geliefert.

Das Năchstliegendste bei meinen Versuchen war, die Granula als lebende Strukturelemente der Săgetiererythrozyten in die Blutbahn von Såugetieren einzubringen. So glaubte ich am ehesten etwaige physiologische oder pathologische Wirkungen erzielen zu können. - $\mathrm{Zu}$ meinen Versuchen dienten mir ausnahmslos ausgewachsene Kaninchen, denen ich jeweils eine frische Granulakultur möglichst rasch in eine Vene des sorgfaltig desinfizierten Ohres injizierte. Ich verwendete $z u$ den Injektionen Granulakulturen, die im Kondenswasser der Röhrchen aufgeschwemmt waren, oder Kulturen, die zuerst in sterilisiertem Brunnenwasser von $37^{\circ} \mathrm{C}$ vorsichtig zentrifugiert, gewaschen und aufgeschwemmt wurden. 
Pathologische. Wirkungen konnte ich in keinem meiner zahlreichen Tierversuch e beobachten. Die Tiere blieben munter, verloren nicht im geringsten ihre gewöhnliche Fresslust, behielten ihr Durchschnittsgewicht usw. - Dagegen ergaben sich bei der Untersuchung des Versuchstierblutes Beobachtungen, die für die $\mathrm{Physiologie}$ des Blutes von' grösster Bedeutung sind.

Gewöbnlich entnahm ich schon am ersten oder zweiten Tag nach der Granulainjektion den betreffenden Tieren nach leichtem Einstich in eine gestaute Ohrvene eine Probe Blut (bei kräftigen Tieren bis zu $10 \mathrm{ccm}$ ). Dieses in engen, gründlich gereinigten und getrockneten Reagenzgläschen aufgefangene Blut wies gegenüber dem in gleicher Weise entnommenen Blut der Kontrolltiere auffallende Veränderungen auf. - Oft beobachtete ich schon sogleich beim Austreten des typisch dunkelroten Venenblutes der Versuchstiere einen jähen Umschlag seiner Farbe ins helle Scharlachrot. Immer aber machte ich diese Wahrnehmung bei dem im Reagenzglas aufgefangenen Blut. Dieses machte niemals den Eindruck von venösem, sondern stets von frisch hellrotem, arteriellem Blut. Das Blut behielt diese hellrote Färbung auch ohne Umschütteln tagelang. Die unter dem Einfluss des Luftsauerstoffes zustande kommende Oxydierung des Venenhämoglobins. in Oxyhämoglobin ging hier immer ganz überraschend schneller; auch ohne etwaiges Schütteln, vor sich als beim Venenblut der Kontrolltiere. Im letzteren Falle konnte, wie schon angedeutet, nur durch Umschütteln des Blutes eine ausgiebigere Überführung in Oxyhämoglobin erreicht werden,

Ferner liess das Blut der Versuchstiere gegenüber dem der Kontrollkaninchen ganz auffallende Unterschiede bezüglich der Gerinnungsverhältnisse erkennen. Wärend das Blut der Kontrolltiere spätestens nach 15 Minuten die typische Gerinnung mit deutlicher Serumauspressung zeigte, die von Stunde zu Stunde zunahm, war die Gerinnungsfähigkeit des Blutes der Versuchstiere entweder vollkommen aufgehoben (besonders nach kurzem, sanftem Umschütteln) oder doch zum mindesten ganz.wesentlich vermindert. Diese stets zu beobachtende Herabsetzung der Gerinnungsfähigkeit kam darin zum Ausdruck, dass sich gewöhnlich nur geringfügige, schleimig-weiche, hellrote Koagula in dem genau gleichfarbigen Blute bildeten. Eine Serumauspressung habe ich in keinem einzigen der zahlreichen Fälle beobachten können. 
Ein leichtes Durchrühren dieser schwach und gleichmässig verdickten Blutmasse hatte zur Folge, dass sie sich bis auf unwesentliche, schleimig-weiche, hellrote Reste wieder in flüssiges Blut auflöste. - Bei der sofortigen mikroskopischen Untersuchung zeigte dieses Blut im Gegensatz zum Blut mit normaler Gerinnung so gut wie keinerlei Fibrinbildung, auch nicht nach Stunden! - Nach verschiedenen Methoden gefarbt, wies es qualitativ wie quantitativ gegenüber dem Normalblut keinerlei nennenswerte Verănderungen -auf. Ehe ich die Folgerungen aus meinen Versuchen für den Gerinnungsvorgang bespreche, möchte ich alle Erscheinungen erörtern, die mit der Sauerstoffaufnahme durch Säugetierblut nach Granulainjektionen in Zusammenhang stehen.

Die Erythrozyten der Versuchstiere zeigen schon kurze Zeit (1-2 Tage) nach der Injektion kultivierter Erythrozytengranula eine gegenüber dem Normalblut der Kontrolltiere deutlich erhöhte Aviditat zum Luftsauerstoff. Diese Folgerung ergibt sich schon aus dem sehr jahen Umschlag der dunkelroten Farbe des Venenblutes in das helle Scharlachrot, wie es nur dem arteriellen Blut zukommt. Diese leuchtend rote Farbe bleibt wăhrend mehrerer Tage unverăndert erhalten. Ich sehe darin die fortgesetzt lebhafte Zellatmung, d. h. die lebhafte Aufnahme von Sauerstoff durch die Erythrozyten des betreffenden Versuchstieres. Das Blut zeigt, solange es seine helle Scharlachfarbe festhălt, in entsprechender Verdünnung bei der spektroskopischen Untersuchung die beiden typischen Absorptionsstreifen des Oxyhämoglobins zwischen der D- und E-Linie des Spektrums. Hier soll darauf verwiesen werden, dass das Blut auch spăter nicht etwa lackfarben wird, denn die Erýthrozyten sind, wenigstens solange sie Oxyhämoglobin führen, gut in ihrer Form erhalten, Fibrinbildung ist nicht festzustellen. - Folgende ebenso einfache wie lehrreiche Versuche demonstrieren sehr anschaulich die grössere Sauerstoffavidităt des Blutes der Versuchstiere gegenüber dem Kontrolltierblut. Ein Reagenzröhrchen von $10 \mathrm{~cm}$ Höhe und einem Durchmesser von ungefähr $2 \mathrm{~cm}$ füllt man bis zur Höhe von $7 \mathrm{~cm}$ mit Brunnenwasser und gibt mit der Pipette soviel Blut eines Kaninchens, dem 24 Stunden vorher eine Granulakultur injiziert wurde, hinzu, dass die Lösung durchsichtig hellkirschrot erscheint. Ein zweites genau gleiches Röhrchen füllt man ebenfalls bis zur Höhe von $7 \mathrm{~cm}$ mit Brunnenwasser und 
fügt soviel Normalblut hinzu, bis auch diese Lösung die gleiche hellkirschrote Farbung wie die erste Blutlösung aufweist. Beide Röhrchen werden sodann zwecks Unterscheidung mit Zeichen versehen und offen bei Zimmertemperatur an einem ruhigen Standort sich selbst überlassen. - Schon bisweilen nach Verlauf von 24 Stunden macht sich eine deutliche Verschiedenheit der Blutverdünnungen in beiden Reagenzröhrchen bemerkbar. Am ehesten beobachtet man sie in dem Röhrchen, welches das Blut des Versuchstieres enthălt: der untere Teil der Flüssigkeitssåule zeigt im Gegensatz zum oberen Anteil mit hellkirschroter Farbe die deutliche, oft etwas getrübte Farbe des venösen Blutes. Die verschiedenartig gefarrbten Flüssigkeitsanteile sind scharf gegeneinander abgegrenzt. Die scharfe Abgrenzung halt sich mitunter bis zur Dauer einer. Woche unverandert, um sich dann zugunsten der venösen Farbung immer mehr und solange nach oben zu verschieben, bis die letzte Spur der hellkirschroten Farbung verschwunden ist. Bei der spektroskopischen Untersuchung zeigt der antere Anteil den breiten, etwas verwaschenen Absorptionsstreifen des reduzierten Hămoglobins, der obere Anteil die bekannten beiden Absorptionsstreifen des Oxyhamoglobins. - An der Blutlösung des anderen Röhrchens kann man unter Umstanden gleichfalls jene markanten Farbenunterschiede beobachten, doch nimmt der untere, venös gefarbte Anteil niemals die Ausdehnung an wie bei der Blutlösung des ersten Röhrchens. Gewöhnlich aber vollzieht sich die Überführung des Oxyhămoglobins in reduziertes Hamoglobin gleichmåssig durch die ganze Flüssigkeitssăule. - Im Gegensatz zur anderen Lösung bilden sich im verdünnten Normalblut meist feinste, spinnwebartige Fibrinfädchen. die ich in der anderen Blutlösung niemals beobachtet habe.

Die Ergebnisse dieser Versuche sind wohl so zu deuten : in beiden Blutlösungen sehen wir die Wirkungen oxydativer Vorgănge, wie sie unter Vermittlung von Oxyhămoglobinlösungen zustande kommen. Am lebhaftesten sind diese Oxydationsprozesse in dem Röhrchen, welches das verdünnte Versuchstierblut enthalt. Die baldige Entstehung der bohen Zone des venösen, blaurot gefarbten unteren Anteils ist nur so zu erklären, dass der oxyhamoglobinhaltige, obere Anteil der Blutlösung das Oxyhamoglobin des vom Luftsauerstoff abgeschlossenen unteren Anteils gleich intensiv reduziert, wie er selbst den ihm zur Ver- 
fügung stehenden Luftsauerstoff an sich reisst. Dass es sich bei der Verschiebung der Grenze zwischen den beiden verschieden gefärbten Blutlösungen zugunsten des blauroten Antejles nicht etwa um rein physikalische Diffusionsvorgänge handelt, sondern tatsachlich und vor allem um kråftigste Oxydationsprozesse des oberen Anteils, erhellt schon daraus, dass die Zone des venösen Anteils bisweilen schon innerhalb $4-5$ Stunden um mehr als das Doppelte an Ausbreitung gewinnt. Erst allmählich verliert auch der obere Anteil der Oxyhämoglobinlösung seine anfänglich erhöhte Oxydationskraft, verhält sich mehr und mehr passiv und wird selbst reduziert. - Das verdünnte Normalblut des Kontrolltieres lăsst die wesentlich intensivere Oxydationsfähigkeit des Versuchstierblutes vermissen; es verfallt gewöhnlich allmăhlich und gleichmässiger der Oxydation durch den Luftsauerstoff sowie der schliesslichen Reduktion durch verschiedene aërobe Fäulnismikroorganismen.

In beiden Versuchen kommt lackfarbiges, oxyhämoglobinhaltiges Blut zur Verwendung, das also so gut wie keine Erythrozyten mehr enthalt, die ihre intakte Form oder auch nur einigermaßen normale Struktur besitzen. W a rburg hat durch seine schönen Untersuchungen gezeigt, dass die Oxydationsfăhigkeit von Zellen, auch von Erythrozyten, dem Grade der Unversehrtheit ihrer Struktur entspricht. Nach dieser Erkenntnis ist die geringe Oxydationsfähigkeit des verdünnten, lackfarbigen Normalblutes durchaus verständlich. Dagegen kann die auffallend erhöhte Oxydationskraft des verdünniten, lackfarbigen Blutes des Versuchstieres nicht erklärt werden, wenn wir sie nicht auf eine bestimmte Wirkung der kultivierten Erythrozytengranula beziehen dürfen, welche wir dem Säugetierorganismus intravenös einverleibt haben.

Bei den gegenüber dem Normalblut der Kontrolltiere wesentlich erhöhten Oxydationsprozessen des Versuchstierblutes handelt es sich um den sehr beschleunigten Ablauf einer spezielleren chemischen Reaktion, der intramolekulären Zellatmung, die durch die Gegenwart der Erythrozytengranula zustande kommt. Die Wirkung dieser Granula, die zur Gesamtblutmenge des Versuchstieres quantitativ in keinem nennenswerten Verhältnis steht, erscheint in Anbetracht ihrer weitschichtigen Verteilung für eine verhältnismässig lange Zeitdauer eine ganz erstaunliche. Wir 
haben also die wichtige Tatsache zu registrieren, dass die kultivierten Erythrozytengranula die Wirkung eines geformten Enzyms entfalten. Der Beweis hierfür lässt sich, abgésehen von der rein physiologischen Wirkung, noch durch folgenden Versuch erbringen, der zudem noch gestattet, die Enzymwirkung der Granula zu spezialisieren. Man wăscht eine etwa 10-14 Tage alte, gut entwickelte Granulakultur mit sterilisiertem Brunnenwasser von $37^{\circ} \mathrm{C}$, ' zentrifugiert vorsichtig, dekantiert das Wasser und schwemmt das so gewaschene Zentrifugat, das nur noch zahlreiche Granula enthalt, in etwas lauwarmem Wasser auf. Gibt man nun einige Tropfen von Wasserstoffsuperoxyd hinzu, so tritt sofort unter lebhaftem Aufschăumen eine Spaltung des Wasserstoffsuperoxyds in Wasser und Sauerstoff ein. Dass der abgeschiedene Sauerstoff aktiv, d. h. sogleich zu kraftiger Oxydation oxydierbarer Substanzen fähig ist, zeigt die mehr oder weniger kräftige Blaufärbung von Guajaktinktur. Soll dieser Versuch beweiskräftig sein, so dürfen nur solche Zentrifugate verwendet werden, welche keinerlei Reste autolysierter oder nekrobiotisch verănderter Erythrozyten, sondern lediglich unsere Granula enthalten. Der Ausfall unseres Wasserstoffsuperoxydversuches lehrt also, dass an die Granula der Erythrozyten $0 x y d a$ se $n$ gebunden sind.

Als wichtigste Ergebnisse meiner Untersuchungen über die physiologischen Wirkungen der Granula ergibt sich folgendes:

1. Die bedeutungsvollsten Strukturelemente der Säugetiererythrozyten sind endozellulär praformierte Granula, welche bei der nekrobiotischen Auflösung der roten Blutzellen frei werden und auf oder in geeigneten Nährsubstraten bei einer Temperatur von $37^{\circ} \mathrm{C}$ zur Vermehrung und zum Massenwachstum angeregt werden können.

2. Die Gesamtheit dieser kultivierten Granula ist den normal gebauten Erythrozyten des strömenden Blutes der Säugetiere in jeder Beziehung durchaus gleichwertig.

3. Die beschriebenen Granula stellen geformte Enzyme im weiteren, geformte Oxydasen im engeren Sinne dar. Sie vor allem ermöglichen unter Vermittlung ihres Hämoglobingehaltes die intrazelluläre Atmung, d. h. die Aufnahme des Luftsauerstoffes, seine Addition an das Hämoglobin (Bildung des Oxyhämoglobins), sowie die Abgabe 
des gebundenen Sauerstoffs in aktiver Form (in statu nascendi). Diese spezielle Funktion kommt den Granulis ebensowohl im Strukturgefüge der lebenden, im Plasma der Saugetiere kreisenden Erythrozyten zu, wie den freien, aus Erythrozytenexplantaten kultivierten Granulis.

Sehr reichlich Oxydasen enthalten bekanntlich die neutrophilen Myelozyten und ihre Abkömmlinge, die neutrophilen polymorphkernigen Leukozyten; geringeren Oxydasengehalt haben die Eiterleukozyten. Die Oxydase ist in allen diesen Zellen an Granula gebunden und aktiviert die Wirksamkeit eines oxydierenden und autolysierenden Fermentes, ferner wahrscheinlich auch diejenige von Lipasen und Diastasen. Ausserdem finden wir Oxydasen in vielen anderen Zellen, namentlich in solchen, die funktionell stark in Anspruch genommen sind, in erster Linie in den verschiedenen Drüsenzellen (Profermente, Zymogene), in den Muskelfasern, in den Ganglienzellen (im Gegensatz zu den Gliazellen!) usw.; auch hier ist die Oxydasereaktion an Granula gebunden. Nach den Anschauungen, die uns die modernen histologischen und chemischen Forschungen an die Hand geben, dürfen wir mit Recht behaupten, dass sich die wichtigsten Stoffwechselprozesse der Zellen in oder an Granulis abspielen. Darauf weist schon die Beobachtung hin, dass die Stărke der Oxydasereaktion und die Anzahl der Granula je nach dem Grade der funktionellen Beanspruchung der sie bergenden Zellen verschieden ist.

Die vielseitige Bedeutung, welche den eben angeführten Granulis für die Stoffwechselvorgănge in der Zelle zukommt, wird vielleicht noch übertroffen von derjenigen der Erythrozytengranula. Sicherlich spielen sie eine grosse Rolle für die auf dem Wege der Diffusion und Osmose vor sich gehende Ernahrung der Erythrozyten, sowie für die Produktion gewisser Immunkörper, besonders der Lysine. Ihre wichtigste Funktion besteht aber zweifellos darin, dass sie die Erythrozyten selbst, sodann alle aktiven Zellen und Gewebe, kurz den gesamten Săugetierorganismus, mit dem zur Ernăhrung und zur Atmung unentbehrlichen Sauerstoff versehen. Die sich in den Erythrozyten abspielenden Oxydationsprozesse sind durchaus nicht einheitlich. Wir wollen sie kurz von dem Standpunkt aus betrachten, dass sie durch Granula vermittelt werden. - Die hămoglobinhaltigen Granula, die wir uns in feinster Verteilung der Erythrozytenstruktur ein- 
gefügt denken müssen, sind zunăchst dazu befähigt, aus der Alveolarluft der Lungen soviel Sauerstoff an sich zu reissen, als seinem Partiardruck entspricht. Durch die Sauerstoffaddition seitens des Hämoglobins entsteht das Oxybämoglobin, eine Peroxydverbindung, welche in ihrem chemischen Verhalten durchaus dem Wasserstoffsuperoxyd analog ist und den Sauerstoff in lockerer Bindung enthält. Seine Abgabe reguliert sich sehr genau nach dem jeweiligen Bedürfnis, seine Verwendung ist sehr verschiedenartig. Ein Sauerstoffanteil dient sicherlich der intramolekularen Zellatmung, d. b. denjenigen Oxydationsprozessen, welche allein das Bestehen und die spezifischen vitalen Vorgänge der Erythrozyten ermöglichen. Ein anderer Sauerstoffanteil des Oxyhămoglobins hat die nicht minder wichtige Funktion, durch die Oxydation organischer Nährstoffe die für das Bestehen des Gesamtorganismus unentbehrlichen Energien (Wärme, Bewegung, Kraftleistungen) $\mathrm{zu}$ schaffen. Der an das Oxyhämoglobin addierte Luftsauerstoff ist zur ausreichenden Verbrennung der organischen Substanzen ohne weiteres nicht fähig; wir wissen, dass die dazu erforderliche Aktivierung des Sauerstoffs durch Oxydasen, welche die Erythrozyten zur Verfügung stellen, vermittelt wird. Meine Untersuchungen haben festgestellt, dass diese Oxydasen an intrazellulare oder freie Erythrozytengranula gebunden sind. - So notwendig einerseits eine kråftige aktive Oxydation für die Erzeugung der erforderlichen Energiemengen des Organismus ist, so notwendig ist es andererseits, dass die Erythrozyten als Trăger und Spender des Sauerstoffs, ebenso die lebenswichtigen anderen Körperzellen vor der allzu intensiven Wirkung der Oxydasen geschützt werden müussen. Es ist überaus wahrscheinlich, dass diesen Schutz besondere Fermente, Antienzyme, Antioxydasen übernehmen, die sogenannten Katalasen, die gleichfalls wie ihre Antagonisten an Granula gebunden sind. Auch für die Abspaltung des inaktiven Sauerstoffs aus dem Oxybämoglobinmolekül, wie er der Unterhaltung der intramolekulären Zellatmung dient, möchte ich an Granula gebundene Katalasen verantwortlich machen. Auch die Katalasen spalten aus dem Wasserstoffsuperoxyd Sauerstoff $a b$, der jedoch inaktiv ist, $d . h$. sich nicht fähig zu intensiverer Oxydation erweist (keine Blaufärbung von Guajaktinktur!). $\mathrm{Ob}$ die Blutoxydasen und Katalasen getrennt an verschiedene oder an die gleichen Erythrozytengranula gebunden sind, lässt sich Archiv f. mikr. Anat. Bd. 92. Abt.. . 
heute noch nicht mit Sicherheit entscheiden; der letztere Fall jedoch gehört zweifellos eher in den Bereich der Möglichkeit. Dies um so mehr, als wir nach Gold manns geistvollen Forschungen über die Vitalfärbung in den vital färbbaren Granulis keineswegs etwa Degenerations- oder Stoffwechselprodukte, sondern präformierte Zellorganula (Arnold, Ribbert u. a.) sehen dürfen, in denen gleichzeitig die verschiedenartigsten biochemischen Reaktionen ablaufen können. Ich erinnere hier nur an die verschiedenen Fermente, an Granula fixiert, in den polymorphkernigen Leukozyten! In gleicher Weise möchte ich auch die bemerkenswerte Beobachtung deuten, dass sich in der isotropen Substanz der quergestreiften Muskulatur an gleicher Stelle vitàl sich färbende Granula und Glykogentröpfchen nachweisen lassen und schliesslich Granula, welche die Oxydasereaktion geben (Gierke).

Für das Verständnis der wichtigsten Lebensprozesse, mit denen sich die Pliysiologie des Blutes befasst, ist es unumgänglich notwendig, sich mit der Frage zu befassen, wie diese erstaunlichen hămatogenen Fermentwirkungen zustande kommen. Die Katalasen und Oxydasen des Blutes wirken auf das Oxyhämoglobin ein, das, wie schon oben erwähnt, als organische Peroxydverbindung in seinem chemischen Verhalten dem Wasserstoffsuperoxyd analog ist. Es wird zum besseren Verständnis meiner weiteren Ausführungen beitragen, wenn wir diejenigen Reaktionen des Wasserstoffsuperoxyds kurz betrachten, welche dieser Analogie zugrunde liegen. Wasserstoffsuperoxyd, rein und wasserfrei dargestellt, sowie vor feinen korpuskularen Verunreinigungen geschützt," ist eine sehr beständige Verbindung. Diese Beständigkeit geht sofort verloren, wenn feinste, feste Partikelchen im Wasserstoffsuperoxyd suspendiert werden; das $\mathrm{H}_{3} \mathrm{O}_{2}$ - Molekül wird rasch in Wasser und Sauerstoff gespalten. Ganz besonders beschleunigt wird die Auslösung und der Ablauf dieser Reaktion durch die Anwesenheit von Katalysatoren in Form fein verteilter Metalle, wie z. B. durch elektrolytisch dargestelltes kolloidales Silber, Gold oder Platin (Platinschwarz). Die katalytischen Wirkungen werden dadurch hervorgerufen, dass der Katalysator entsprechende Teilchen des Absorptionsmediums auf seiner durch die kolloidalen Metallteilchen enorm vergrösserten Oberfläche verdichtet. Der Katalysator steht in der Regel quantitativ in 
keinem nennenswerten Verbăltnis zur entsprechenden katalytischen Reaktion und den durch sie gelieferten Energiemengen. Der katalytisch aus dem Wasserstoffsuperoxyd abgespaltene Sauerstoff ist an sich ziemlich inaktiv, er kann z. B. keine Blaufärbung einer mit Stärkekleister versetzten Jodjodkaliumlösung verursachen. Diese Blaufärbung tritt sofort ein, wenn man etwas Ferrosulfat zusetzt, das dem Jodjodkalium gegenüber als Sauerstoffüberträger wirkt und zwei Jodatome in Freiheit setzt, die sich dann mit der Stărkelösung zur blaugefarbten Jodstărke verbinden. Die einfache katalytische Sauerstoffabspaltung aus dem Wasserstoffsuperoxyd entspricht der Wirkung der organischen Katalase, die wirksame Sauerstoflübertragung durch Ferrosulfat dem Effekt einer Oxydase. - Zwischen den anorganischen Katalysatoren und den organischen Enzymen und Fermenten bestehen bekanntlich in mancherlei Hinsicht auffallende Ähnlichkeiten. Zunachst ist $\mathrm{zu}$ betonen, dass beide selbst in geringsten Mengen noch bedeutende katalytische Wirkungen entfalten. ohne selbst irgendwelche betrachtliche Veränderungen zu erleiden. So beschleunigt $z$. B. $1 \mathrm{~g}$-Atom Platin in 70000000 Litern Wasser noch sehr wesentlich die Zersetzung von Wasserstoffsuperoxyd; $0,0000000001 \mathrm{~g}$ Vanadin beschleunigt noch aufallend die Gerinnung der Milch ( $\mathrm{R} \mathrm{j} \mathrm{b}$ e t). Lösungen anorganischer Katalysatoren und organische Fermente sind kolloidaler Natur; sie haben ein Temperaturoptimum ihrer katalytischen Wirksamkeit, die bei Einwirkung höherer Temperaturen oder durch gewisse Gifte (z. B. 10-6 Mol HCN) in stärkster Verdünnung vernichtet wird.

Die einfache katalytische Zerlegung des Wasserstofisuperoxydmoleküls in inaktiven Sauerstoff und Wasser durch Schwebefallungen kolloidaler Metalle ist als ein rein physikalischer Vorgang aufzufassen, bei dem die dissoziative Spaltung des Adsorptionsmediums durch die Adsorption an eine enorm vergrösserte Oberfläche zustande kommt. Im Gegensatz bierzu kommen für die Abspaltung des akiven, d. h. direkt zur Oxydation fühigen Sauerstofls aus dem Wasserstoffsuperoxyd $z$ we i verschiedene Wirkungskomponenten in Betracht:

1. eine rein physikalische, bedingt durch Adsorption an enorm vergrösserten Wirkungsobertächen,

2. eine elektiv-spezifische, die in unserem oben beschriebenen Wasserstoftsuperoxyd-Versuch in der Affini- 
tăt des Ferrosulfatmoleküls zum Jodkaliummolekül und zum inaktiven, katalytisch abgespaltenen Sauerstoffatom zum Ausdruck kommt. -

Ohne Zweifel spielt für die Wirkung der Oxydationsfermente ebenso wie für diejenige anorganischer Katalysatoren die Adsorption an stark vergrösserte Wirkungsoberflachen eine sehr bedeutende Rolle. Das geht schon daraus hervor, dass die ${ }^{\bullet}$ Wirkung der durch Katalasen oder Oxydasen ausgelösten Oxydationsprozesse quantitativ in einem ganz bestimmten Verhăltnis zur Ausdelınung der Oberflachen steht, auf die sich die Wirkung jener Oxydationsvorgange erstreckt. Ich verweise hier abermals auf Warburgs Arbeiten. Er hat z. B. nachgewiesen, dass die Oxydationsgeschwindigkeit von seiten unbefruchteter Seeigeleier mit noch wenig komplizierter Struktur nur unmerklich beeinflusst wird, dass sich dagegen die Geschwindigkeit der Oxydationsprozesse in. den gleichen Eiern wahrend der Befruchtung ganz enorm erhöht. Diese Steigerung ist sicherlich auf die Oberflächenvergrösserung zu beziehen, die durch die Ausbildung der Keimzellenfurchung zustande kommt. Die gleiche, intensive Beschleunigung der Oxydationsprozesse lässt sich schon in den ersten Stadien der Eibefruchtung feststellen; sie ist hier auf die Ausbildung komplizierterer Strukturen zurückzuführen, die wie die Spiremknăuel, die Chromosomen und Aktinosphären den Oxydationsvorgăngen stark vergrösserte Wirkungsoberflachen darbieten. - Dass auch für die Wirkung der Oxydationsfermente der Gewebe und des Blutes der rein physikalische Vorgang der Adsorption von grösster Bedeutung ist, darauf weist schon die interessante Tatsache hin, dass in der Struktur aller lebenswichtigen Organe das Prinzip vergrösserter Wirkungsoberflachen gewahrt ist, in dem der Adsorption des Leben anregenden und erbaltenden, sowie die Ernahrung vermittelnden Sauerstoffs die denkbar zweckmăssigsten Bedingungen gegeben sind. Man denke hier an die enorm vergrösserte Atmungsoberflăche der Lungen, wie sie durch die überaus zahlieichen Alveolen und Interalveolarsepten bedingt ist, und die um ein beträchtliches Vielfaches die gesamte aussere Körperoberflăche übertrifft. Weitere Beispiele stellen der gesamte Drüsenapparat des Körpers mit seinen Einbuchtungen, Lappen, Lappchen und seinem weitverzweigten Kanalchensystem, das Gehirn mit seinen kunstvollen Windungen und Einbuchtungen, 
der Darmtraktus mit seinen Haustren, zahlreichen Falten und zahllosen Zotten und Krypten, das vielfach gefaltete Peritoneum mit seiner erstaunlichen Adsorptions- und Resorptionskraft, der Muschelapparat der Nase u. a. m. Dass die Funktionen dieser Organe, deren Strukturen schon makroskopisch dem Prinzip der Oberflăchenvergrösserung aufs weitgehendste Rechnung tragen, nur durch ein Zirkulationssystem mit entsprechend gleichem Prinzip gewabrleistet werden können, ist selbstverstandlich. Vielleicht nirgends ist im tierischen Organismus das Prinzip der vergrösserten Wirkungsoberflachen so weitgehend durchgeführt wie in der Blutverteilung und Blutversorgung. Von der Aorta ascendens an vergrössert sich bekanntlich das arterielle Gebiet durch stetige Abgabe von Seitenassten und -astchen derart, dass sich im Kapillargebiet der Querschnitt des Strombettes etwa um das 700 fache des ursprünglichen Querschnittes vergrössert hat (Vieror d t). Dieser enormen Vergrösserung des Strömungsquerschnittes entspricht natürlich auch eine bedeutend erhöhte Wirkungsintensitat chemiscber, vor allem oxydativer Prozesse in der Gesamtblutmasse, die auf so grosse Oberflachen verteilt ist. Sehr wirksam wird die Oxydationsoberflăche des Blutes dadurch vergrössert, dass die Oxydationsvorgange an die Hamoglobin führende Substanz der überaus zahlreichen regelmåssig- und gleichgeformten Scheibchen der Erythrozyten gebunden sind. Ferner und endlich erreicht die Masse der Erythrozyten den grösstmöglichen Grad ihrer chemischen und physikalischen Wirksamkeit durch ihre besondere Struktur, deren wesentlichste und difterenteste Elemente ich in den oben ausführlich beschriebenen Granulis sehe. Die Granula sind ihrer chemischen Natur nach organische Kolloide, die wir uns in den normalen Erythrozyten in ausserst feiner und dichter Verteilung vorstellen müssen, jedenfalls in weit feinerer Verteilung, als wir sie mit Hilfe der verschiedenen Farbemethoden wahrnehmen können. Die Grösse, die Zahl und die intrazellulăre Lagerung der Granula wechseln je nach den verschiedenen Phasen der physiologischen oder pathologischen Lebensvorgange, unter deren Einfluss jeweils die roten Blutzellen stehen. - Wie ich schon erwahnte, sind die Erythrozytengranula hămoglobinhaltig. Die Annahme Abderhaldens, dass das sogenannte „Funktionseisen" (M. B. Schmidt) möglicherweise kolloidal in den Erythrozyten gelöst sei, hat vieles für sich. Ich 
halte es für sehr wahrscheinlich, dass sich das Funktionseisen im Hamoglobin der intra- und extrazellulären Granula in kolloidaler Lösung befindet. So ist vielleicht die Unmöglichkeit $\mathrm{zu}$ verstehen, jenes Eisen durch chemische Reaktionen nachzuweisen. - A bderhalden hat die Wichtigkeit der Kolloide für den gesamten Stoff- und Gaswechsel der Zelle ganz besonders hervorgehoben. Wohl an keinem anderen Beispiel kann die Bedeutung der Kolloide so lehrreich gezeigt werden, wie an den roten Blutzellen. Wenn man das System verfolgt, nach dem die Blutzirkulation dem Prinzip vergrösserter Wirkungsoberflachen bis an die denkbar möglichste Grenze Rechnung trăgt, gewinnt man eine Vorstellung vom Zustandekommen jener gewaltigen Oxydationsvorgånge im Săugetierorganismus, die sich zu einem betrăchtlichen Teil sogar auf rein physikalischer Basis abspielen. Wie sich beim Sauerstoff spendenden Zirkulationsapparat jenes Energien scbaffende Prinzip bis auf die feinsten, ja ultramikroskopischen Strukturelemente der Erythrozyten erstreckt, so ist es auch bei den Sauerstoff empfangenden Geweben und Organen mit ausserster. Gleichwertigkeit gewahrt. Den sprechendsten Beleg hierzu gibt der ausserst komplizierte Bau der verschiedenen Drüsenzellen. Es würde mich zu weit führen, hier Einzelheiten auch nur zu streifen. Soviel steht fest, dass auch in der Struktur der Drüsenzellen durch zahlreiche verschiedenartige Granula oder Mitochondrien das Prinzip der vergrösserten Wirkungsoberflächen wiederum bis aufs ausserste zur Geltung kommt. Ich brauche kaum hervorzuheben, dass wir auf Grund neverer histologischer (M. He id e $\mathrm{nhain}$ ) und vitalfärberischer (Arnold, Gold man $n$ u. a.) Untersuchungen in den Drüsengranulis keinesfalls nur Sekretkörnchen oder geformte Profermente usw. zu sehen haben, sondern dass es sich bei den meisten um Mikrosomen, um Plasmosomen handelt, denen Leben, Wirken und sogar Regenerationsfăhigkeit zukommt.

Sach meinen obigen Ausführungen ist die Wirkung der Katalasen dem Effekt anorganischer Katalysatoren analog. Bei beiden ist die rein physikalische Wirkungskomponente, die Adsorption an enorm vergrösserte Oberflăchen, das Ausschlaggebende. So ist leicht einzusehen, dass das Prinzip vergrösserter Oberflächen, wie es im Blutzirkulationsapparat verkörpert ist, besonders die Abspaltung des inaktiven, allein dem Zellenleben dienen- 
den Sauerstoffs durch Katalasen in hervorragendem Maße begünstigt. Auf Oberflachenwirkung zwischen Adsorbens und Adsorbendum ist z. B. grösstenteils die Katalysenwirkung der Milch (Analogie mit dem Blute!). zu beziehen (zahllose Fett- und Kollostrumgranula). Nach den Untersuchungen Batell is und Sterns übt auch das Lebergewebe, nach den Arbeiten Spitzers auch das Milzgewebe energische Katalasenwirkung aus; ob. diese Katalasen nicht etwa mit denen des Blutes identisch sind, darüber lasse ich die Entscheidung offen.

Dass auch die Wirkung der Blutoxydasen durch vergrösserte Oberflăchen, wie sie die Blutverteilung im grossen und die granuläre Struktur der Erythrozyten im besonderen, ganz wesentlich gefördert wird, ist sicher, ebenso gewiss aber kann ihre Wirkung ohne eine spezifisch-elektive Komponente nicht zustande kommen. Die rein physikalische Komponente ist also durch das Prinzip vergrösserter Oberfächen gegeben, der spezifische Anteil durch die elektive Aktivierung des katalasenartig abgespaltenen Sauerstoffs. Man darf mit Recht. die Oxydasen spezifische Fermente nennen, deren Spezifită je nach den normal oder pathologisch ablaufenden Stoffwechselprozessen der Zellen und Gewebe verschieden ist. Man denke beispielsweise an die weitgehende Oxydierung von Eiweiss, Fetten und Kohlehydraten (Traubenzucker!) im normalen Organismus und gegensätzlich dazu an die mehr elektive Oxydierung von Eiweiss und Fetten bei Störungen, wie sie dem Diabetes mellitus zugrunde liegen! Jedenfalls liegt auch eine der Hauptfunktionen der Oxydasen in der Aktivierung Fett, Eiweiss und Kohlehydrat spaltender Enzyme; ebenso spielen sie wahrscheinlich für die Reversibilităt der verschiedenen Enzymwirkungen und damit für die Synthese eine nicht zu unterschătzende Rolle. Darauf deuten $u$. a. die Lntersuchungsergebnisse von Doyon und Dufourt, die bei Unterbindung des arteriellen Blutzuflusses zur Leber eine auffallende Verminderung der Harnsäuremenge beobachten konnten, wảhrend Pfortaderligatur ohne nennenswerte Beeinflussung der Harnsäurebildung blieb. Sebr wichtig und beweisend sind auch die Versuche von Horbaczewski, der durch Digestion von Milzpulpa und Lebergewebe mit sauerstoffhaltigem Blut eine Harnsäuresynthese erzielt hat. Auf Einzelheiten dieser höchst komplizierten und noch vielfach ungeklarten Vorgange kann ich hier keinesfalls ein- 
treten, ich kann nur auf die bedeutendsten diesbezüglichen Arbeiten $\mathrm{Abderhaldens}$ verweisen.

In den letzten Abschnitten vorliegender Abhandlung versuchte ich darzulegen, welche vielseitige Bedeutung den hămatogenen, an Erythrozytengranula gebundenen Oxydationsvorgăngen für das normale Blut und den normalen Saugetierorganismus zukommt. Die Überzeugung von dieser Bedeutung, ja von der Lebenswichtigkeit dieser Granula wird sich noch mehr festigen, wenn wir ihre Einwirkung auf einen Saugetierorganismus kennen lernen, bei dem die Funktion des Blutes durch akute Schadigungen schwer beeintrachtigt ist. Es handelt sich hier vornehmlich um die Beeintrachtigung der Atmung und des Lebens durch akute Anamien und giftige Blutgase.

Bei der posthämorrhagischen Anamie beherrscht das Krankheitsbild eine Symptomatologie, die durch eine starke Reduzierung der Blutmenge bedingt ist; ihr liegt eine starke Verminderung der Erythrozytenanzahl und damit eine pathologische, vom Organismus an sich nicht zu erganzende Beeintrăchtigung der Oxydations- und Atmungsoberflăche zugrunde. Sehr haufig treten bei der posthamorrhagischen Anamie noch ausserdem Erscheinungen von seiten des Zirkulationsapparates auf, die sich besonders in lebensbedrohlicher Blutdrucksenkung geltend machen. Bei der akuten Anamie handelt es sich also um pathologische Verănderungen physikalisch-chemischer Natur. Im Gegensatz hierzu ist bekanntlich die wichtigste Blutgasvergiftung, die durch Einatmung von Kohlenoxyd hervorgerufen wird, allein durch anormale chemische Prozesse bedingt, vornehmlich durch die im Verhăltnis zum Sauerstoff etwa 200 fach grössere Affinităt des Kohlenoxyds zum Hamoglobinmolekül. In beiden Fallen schwerer Schadigung der Blutfunktion wendet man mit auffallendem Erfolg die Transfusion artgleichen Blutes an. Die oft lebensrettende Wirkung ist gewöhnlich die gleiche, ob dieser Eingriff direkt mittels Gefässnaht nach Carrel, Sauerbruch u. a., oder indirekt durch Injektion ausgeführt wird, ob das Blut des Spenders mit allen seinen Formelementen oder defibriniert zur Verwendung kommt. Bei der akuten Anamie ist die lebensrettende Wirkung obne weiteres dann verständlich, wenn grössere Mengen Blutes transfundiert werden, denn einerseits wird die ausgiebigere Blutmenge wieder normale Blutdruckwerte und damit 
normale Zirkulationsverhaltnisse schaffen, andrerseits bleiben nach neueren Forschungen die meisten der in sehr grosser Anzahl transfundierten Erythrozyten erbalten und befähigen den lufthungrigen Organismus wieder $\mathrm{zu}$ ausreichender Oxydation und Atmung. Dagegen steht in nicht wenigen Fallen der erfreuliche Erfolg einer "Blutüberpflanzung“ in keinem entsprechenden Verhăltnis zur infundierten Blutmenge. Oft leisten schon $5-10 \mathrm{ccm}$ Blut Erstaunliches. Ich verweise hier auf die interessanten und praktisch ausserst wichtigen Mitteilungen, die kürzlich Wederbake uber seine Beobachtungen bei der indirekten Bluttransfusion veröffentlicht hat. Wederhake sieht in dem transfundierten Blut das wirksamste Herz- und Atmungsreizmittel, das ihm in Notfallen zur Verfügung stand. Die Erythrozyten treten nach Infusion grösserer Blutmengen sofort in Funktion und beseitigen den Lufthunger wie mit einem Schlage. Bei akuten grösseren Blutverlusten ist nach $W$ ederhake die Überpflanzung von menschlichem Blut auch dann noch indiziert, wenn bereits die Atmung steht und die Pupillen reaktionslos sind. Bedingung sei nur, dass das Herz durch künstliche Atmung und Stossen an die Herzgegend $z u$ einigen Kontraktionen angeregt werden könne. Es ist kaum anzunehmen, dass die sehr hăufig beobachtete rasche, fast augenblickliche günstige Beeinflussung eines akut-anămischen Zustandes nach Transfusion einer so geringfügigen Menge von etwa 5-10 ccm Blut durch eine gleich rasche Regeneration von Erythrozyten in den Blutbildungsstatten zustande kommt. Dagegen ist es mehr wie wahrscheinlich, dass bei jeder erfolgreichen und lebensrettenden Bluttransfusion zum mindesten katalytische Vorgange im Spiele sind. Ich bin der Ansicht, dass diese Wirkung hauptsăchlich den Erythrozytengranulis als energischen Sauerstoffüberträgern zu verdanken ist. Schon die Tatsache, dass Oxydasen an die von mir beschriebenen Granula gebunden sind, spricht dafür, nicht minder auch die Tierversuche, die ich im folgenden Abschnitt kurz erwahnen will. In gleicher Weise spricht für eine energische katalytische Sauerstoffübertragung durch infundierte rote Blutzellen die namentlich in letzter Zeit wiederholt gemachte Beobachtung, dass der Erfolg einer Bluttransfusion durch gleichzeitige oder bald nachfolgende Kollargolinjektion (!) wesentlich gefördert wurde ( $M$ u rath u. a.). Also ein gleichgerichtetes Zusammenwirken 
organischer und anorganischer Katalysatoren! Die Temperatursteigerungen, die sich in der Regel im Anschluss an direkte Bluttransfusionen beobachten lassen, möchte ich auf Rechnung unverhalltnismässig beschleunigter Oxydationsvorgange setzen. Die endgültige Wirkung einer Bluttransfusion, welche die katalytische Reizwirkung der infundierten Erythrozyten überdauert, ist natürlich auf die Schaffung ansreichender Oxydations- und Atmungsoberflăchen durch ausgiebige multiple Regeneration roter Blutzellen $z u$ beziehen. Wir gehen kaum fehl mit der Annahme, dass für die Auslösung und wirksame Fortfuhrung dieser Regeneration ebenfalls katalytische Wirkungen unserer Erythrozytengranula verantwortlich gemacht werden müssen, um so mehr, als wir durch Engelmanns und Verworns bekannte Versuche über die Bedeutung des Sauerstoffs für das Leben und die Regeneration der Zelle unterrichtet sind.

Bei der Kohlenoxydvergiftung entsteht - wie schon erwahnt - die schwere Atmungsinsuffizienz dadurch, dass das Kohlenoxyd mit dem Hamoglobin, zu dem es eine weit grössere Affinităt besitzt als Sauerstoff, sich zu Kohlenoxydbămoglobin verbindet, eine Verbindung, die durch "Massenwirkung " von seiten des Sauerstoffs wieder gesprengt werden kann. Diese Massenwirkung kann nur dann erfolgreich in Funktion treten, wenn man dem CO-vergifteten Organismus sobald als möglich, unter energischer Zuhilfenahme künstlicher Atmung, konzentrierten und unter gewissem Druck stehenden Sauerstoff zuführt. Bessere Erfolge verspricht - ebenfalls nur bei rascher Anwendung die sogenannte „deplethorische Transfusion“, bei der ein Teil des vergifteten Blutes entzogen und durch frisches, funktionskräftiges Blut ersetzt wird. Der Erfoly dieser Transfusionsart, der sich ohne weiteres aus den chemischen Verbăltnissen der Kohlenoxydvergiftung erklărt, veranlasste mich, die Wirkung meiner kultivierten Erythrozytengranula am $\mathrm{CO}$-vergifteten Tierorganismus zu studieren. Gleichaltrige, ausgewachsene Kaninchen wurden unter einen gut abgedichteten Glasrezipienten gesetzt, dem gleiche und genan zugemessene Quantitaten Leuchtgas mit Hilfe eines Pneumothoraxfüllapparates zugeleitet wurden. Je nach dem sehr wechselnden Kohlenoxydgehalt und der Menge des zugeführten Leuchtgases entwickelten sich mehr oder weniger rasch die typischen Vergiftungserscheinungen: Unruhe, Muskelschwăche, 
Lähmung vornehmlich der hinteren Extremitäten, klonische Zuckungen, vertiefte, verlangsamte und schnappende Atmung, schliesslich Atmungsstillstand. Die Tiere wurden im Zustand völligen Atmungsstillstandes und maximal erweiterter Pupillen aus dem Rezipienten genommen. Bei den einen Tieren wurde leichte künstliche Atmung eingeleitet, den anderen injizierte ich rasch in eine der paralytisch stark erweiterten Ohrvenen das Material von 1-2 etwa 14 Tage alten und im Kondenswasser aufgeschwemmten Kulturen explantierter Erythrozyten, die sehr reichlich Granula enthielten; ausserdem wurde auch bei diesen Tieren leichte künstliche Atmung angewendet. Die nicht behandelten Kaninchen blieben teils leblos, teils erholten sie sich langsamer als diejenigen, welchen die Erythrozytengranula injiziert worden waren. Besonders deutlich zeigte sich die wiederbelebende Wirkung der Granula in einem Falle, wo es sich um ein CO-vergiftetes Tier handelte mit völligem Atmungsstillstand, maximal erweiterten Pupillen und bereits trockener und glanzloser Kornea, in so völlig leblosem Zustand, dass eine Wiederbelebung kaum mehr möglich schien. Neine Versuche wurden leider in letzter Zeit stark beeinträchtigt und bald völlig unmöglich gemacht durch die immer mehr 'wechselnde Zusammensetzung des Leuchtgases und seinen immer ungleichwertiger gewordenen Kohlenoxydgehalt. - Der günstige Erfolg, die wiederbelebende Kraft des transfundierten Blutes oder injizierter Granulakulturen auch in Fällen, wo der Saugetierorganismus bei akuter Anämie oder nach intensiver Kohlenoxydvergiftung hart an der Grenze zwischen Leben und Tod steht, ist zweifellos der katalytischen Wirkung der wichtigsten Strukturelemente der Erythrozyten - ihren Granulis - zuzuschreiben.

Die Intensität katalytischer Oxydationsvorgänge, deren wiederbelebende Kraft nach der Bluttransfusion oder nach Injektion kultivierter Blutexplantate auf die Erythrozytengranula zu beziehen ist, wird in den normalen Erythrozyten des kreisenden Blutes nicht erreicht. Hier wird die Wirkung der Oxydasen durch bestimmte Katalasen und andere Antienzyme gedämpft und in Schach gehalten. Es handelt sich hier sicherlich um komplizierte gegenseitige Regulierungsprozesse und Korrelationen, die an diejenigen bestimmten Strukturen gebunden sind, welche allein die Normalfunktion und die Erhaltung der roten Blutzellen be- 
dingen, d. h. ihren Gleichgewichtszustand in morphologischer und funktioneller Hinsicht. Werden diese korrelativen Regulierungen irgendwie durch anormale innere Einwirkungen (z. B. durch Toxine, unzureichende Ernăhrung usw.) oder durch gröbere Einflüsse ausserer Natur geschadigt, so können unter Umstanden die an differente Strukturelemente - z. B. an Granula - gebundenen Krafte in ihrer ursprünglichen Wirkung ungehemmt zur Entfaltung kommen. Ich verweise hier auf die diesbezüglichen Arbeiten von Virchow, Weigert und Orth. Jene Gleichgewichtsstörungen geben vielfach Veranlassung zur A uslösung autolyt ischer Prozesse innerbalb oder ausserhalb des tierischen Organismas. Bei der Phosphorvergiftung und der akuten gelben Leberatrophie spielt bekanntlich die Autolyse innerhalb des lebenden Organismus eine bedeutsame Rolle und macht sich besonders im Abbau der Leberzellen zu Aminosăuren (Leuzin, Tyrosin usw.) und Hexonbasen (Lysin, Arginin usw.) geltend. Ein Beispiel für die Autolyse ausserhalb des tierischen Organismus liefert das Verhalten der Hefezellen, die auf stickstoffreien, erschöpften Năhrsubstraten ihr Enzym zur eigenen Dekomposition und Dekonstruktion verwenden. Bei der Auflösung der Erythrozyten durch die Einwirkung des hypotonischen Kondenswassers wird der intrazellulare Gleichgewichtszustand auf jede Art gestört und den Granulis die Möglicbkeit gegeben, ihre Fähigkeiten nach jeder Richtung hin zu entfalten. So ist es ihnen denn auch möglich, durch Schaffung vergrösserter Wirkangsoberflăchen die an sie gebundenen Oxydationsvorgănge noch enorm zu potenzieren.

Es ist wohl angezeigt, hier mit einer der wichtigsten Oxydationsstörungen des Săugetierorganismus, mit dem Fi eber, sich kurz zu befassen. Die Lehre vom Fieber entbehrt leider heute noch sehr der wünschenswerten Eindeutigkeit und Einstimmigkeit der Auffassungen. Darüber jedoch sind gewiss såmtliche Autoren einig, dass dem Fieber Störungen im Sinne einer Steigerung der normalen Oxydationsvorgănge zugrunde liegen, die zu einer übernormalen Eiweisszersetzung auf Grund autolytisch-hydrolytischer Spaltungen im lebenden Organismus führen. $\mathrm{Da}$ das Blut wie kein anderes "Gewebe" die hauptsăchlichsten Oxydationsvorgănge vermittelt, so ist es wahrscheinlich, dass derartigen Oyxdationsstörungen, wie sie beim Fieber vorliegen, auch gewisse V'erånderungen morphologischer oder physiologischer Art 
entsprechen werden. Fast alle Forscher haben als ståndige Begleiterscheinung lăngere Zeit andauernder Fieberzustănde eine Oligozythămie feststellen können. Diese Verminderung der roten Blutzellen ist auf eine verschiedengradige Zerstörung von Erythrozyten zurückzuführen. Darauf weist $u$. a. schon die oft zu registrierende wesentliche Zunahme von Hämosiderin in Knochenmark, Milz und Leber hin; zudem möchte ich die von verschiedenen Seiten mitgeteilte Eindickung des Gallenblaseninhaltes, ebenso mit Lö wit die vermehrte Ausscheidung von Kalisalzen, auf Rechnung anormal vermehrter Erythrozytenzerstörung setzen. Jener Oligozytbămie entspricht gewöhnlich auch eine nachweisbare Abnahme des Hämoglobingehaltes. - Die physiologischen Verănderungen, die das Blut wăhrend lănger dauernder Fieberzustånde charakterisieren, sind teils chemischer, teils physikalischer Natur. Es bestehen gewöhnlich Verminderung der Alkaleszenz und verminderter Kohlensauregehalt des Blutes; Azeton, Azetessigsäure, $\beta$-Oxybuttersaure und flüchtige Fettsăuren können im Harn auftreten. Auffallender sind die Verănderungen, welche den Gerinnungsprozess betreffen. Die diesbezüglichen Angaben sind nicht ubereinstimmend, doch überwiegen die Beobachtungen, die eine deutliche Verminderung, ja in gewissen Fallen eine völlige Aufhebung der Gerinnungsfăhigkeit besonders bei akuten Fieberprozessen feststellten. Ganz besonders bemerkenswert finde ich die Angabe von $\mathrm{Lub}$ a r sch, dass in gewissen Phasen der aktiven Tetanusimmunisierung die Gerinnungsfähigkeit des Aderlassblutes vermindert sei; und dass dieses Blut auch nach langerem Stehenlassen eine auffallend geringe Ausbeute an ausgepresstem Serum liefere. - Die Begleiterscheinungen pyrogen erhöhter Oxydationsvorgănge : Zerstörung roter Blutzellen. wesentlich verminderte, unter Umstănden aufgehobene Gerinnbarkeit des Blutes und auffallend geringfügige Serumauspressung bei der Gerinnung zeigen mit den oben beschriebenen Blutveranderungen meiner Versuchstiere nach Injektion kultivierter Erythrozytengranula die grösste Ähnlichkeit. Diese Gemeinsamkeit der Erscheinungen erlaubt uns, sowobJ auf eine Gemeinsamkeit ihrer Verursachung zu schliessen, als auch wechselseitige Schlüsse über Genese und Wirkung des Fiebers und das Wesen der Gerinnung zu ziehen. Die gemeinsame Ursache jener Erscheinungen muss zweifelsohne in anormalen Oxydationsvorgăngen 
gesucht werden, die an die prăformierten Granula der unversehrten Erythrozyten oder an die freien Erythrozytengranula gebunden sind. Diese Annahme darf um so weniger abgewiesen werden, als wir experimentell durch Injektion kultivierter Erythrozytengranula am Blut der Versuclistiere den ganzen Komplex der besprochenen auffalligen Erscheinungen hervorrufen können. Für die angedeuteten chemischen und physikalischen Blutveranderungen bei lănger andauernden Fieberzuständen sind anormal gesteigerte Oxydationsvorgange ebenso wie anormal reduzierte Oxydationsprozesse verantwortlich zu machen. Dafür sprechen die Resultate der Gaswechseluntersuchungen verschiedener italienischer und französischer Autoren, die eine entsprechende direkte Beziehung zwischen der Fieberhöhe und der Grösse des Sauerstoffiverbrauches nicht feststellen konnten. So haben z. B. Arloing und Laulasnié nachgewiesen, dass bei Diphtheriefieber eine gegenüber der Norm erhebliche Verminderung der Oxydationsvorgănge bestehen könne. In den primăr nutritiv, bakteriell oder toxisch geschădigten Erythrozyten mit allgemein verminderter Resistenz treten wahrscheinlich zunachst gesteigerte $0 x y$ dationsprozesse auf, welche sodann unter Umstănden zu einer mehr oder minder ausgedehnten Dekomposition und Destruktion zahlreicher roter Blutzellen unter Vermittlung autolytischer Vorgange führen. Im gleichen $\mathrm{MaBe}$, wie die Struktur der Erythrozyten und damit ihre Funktionen autolytisch geschadigt werden und vor allem die Sauerstoff spendende Oberfiàche der Erythrozyten verringert wird, erfahren auch die Gewebe und Organe des Organismus Störungen ihres vitalen Gleichgewichtes und ihrer Funktion. Eine derartige Schadigung finden wir besonders an denjenigen Organen, die als Sauerstoffempfänger nach dem gleichen Prinzip ausgiebig vergrösserter Wirkungsobertlachen gebaut sind wie der Sauerstoff spendende Apparat der Blutzirkulation. Hier kommen vor allem die Drüsen in Betracht. Ich verweise hier auf die im Fieber verminderte Sekretion der Speicheldrüsen, der verschiedenen Magen- und Darmdrüsen, ferner auf die pyrogen eingeschränkte Milch- und Talgdrüsensekretion. - Die verminderte Alkaleszenz des Fieberblutes ist darauf zurückzuführen, dass sich hauptsăchlich unter dem Einfluss der eben erwähnten autolytischen Zersetzungen, aus dem Material der Erythrozyten und anderer unzureichend mit Sauerstoff versorgter Körperzellen 
organische Säuren (z. B. Milchsăure) bilden, welche zum Teil die Kohlensăure der Blutalkalien substituieren. Bei hochgradigerem Erythrozytenzerfall infolge ungenügender Sauerstoffzufuhr bezw. Sauerstoffbildung kann es, wie schon erwahnt, zur Ausscheidung von Azeton, Azetessigsäure u. a. durch den Harn kommen. Trotz der verschiedenen Theorien ist der Gerinnungsvorgang auch beute noch nicht vollkommen aufgeklart. Ich halte mich hier nur kurz an einige Tatsachen, welche mir am ehesten eine Lösung dieses Problems versprechen. Nach den neuesten Untersuchungen (B ord e t, D e l a n g e, Z a ck) kommt den Lipoiden des Blutplasmas eine hervorragende Bedeutung für die Blutgerinnung $\mathrm{zu}$. Namentlich $\mathrm{Zack}$ hat die interessanten Tatsachen festgestellt, dass Entfettung des Plasmas oder fermentative Spaltung der Plasmalipoide den Gerinnungsprozess stark hemmen oder überhaupt nicht zustande kommen lassen, und dass die so eingebüsste Gerinnungsfahigkeit durch Zusatz lipoidartiger Stoffe regeneriert werden könne. Stuber hat dargetan, dass der Verlust der Gerinnungsfähigkeit des Blutes in jenen Fallen nicht etwa speziell auf eine spezifische Wirkung des Lezithins zurückzuführen sei, sondern auf den Verlust der verschiedenen Neutralfette. Alle diese Untersuchungen legen den Gedanken nahe, dass die verschiedengradige Beeintrăchtigung der Gerinnbarkeit des Fieberblutes auf fermentative Spaltungen von Plasmafettstoffen, ausgehend von autolytischen, zellulăren Zerfallsprodukten, zu beziehen sind. Die Beeintrăchtigung oder Aufhebung der Blutgerinnung, wie ich sie am Blute der mit kultivierten Erythrozytengranulis behandelten Kaninchen beobachten konnte, lassen sich genetisch vielleicht ebenso, d. h. gleichfalls durch fermentative Fettspaltungen im Plasma erklarren. Diese Auffassung ist wohl kaum unberechtigt, da wir besonders auch nach Stubers Untersuchungen die Bedeutung der quantitativen Verhaltnisse für fermentative und ahnliche Prozesse (Agglutination usw.) kennen, und auch deshalb, weil wir in den kultivierten Erythrozytengranulis Träger kräftiger Oxydationsvorgănge sehen gelernt haben.

Blutgerinnung und gewisse Immunprozesse haben bezüglich ihrer Entstehung und ihres Wesens mancherlei Ähnlichkeiten miteinander. So kommt nach den Forschungen der letzten Jahre den Fettstoffen nicht nur für den Gerinnungsvorgang, 
sondern auch für manche Immunprozesse die gleiche Bedeutung zu. Beide Gebiete haben gleiche Grundlagen und lassen sich durch Analogien miteinander verknüpfen. Für den Vorgang der Blutgerinnung sind Fermentwirkungen das Ausschlaggebende. Diese Fermentwirkungen sind zum grössten Teil organisch- oder anorganisch-katalytischer Natur und lassen sich, sogar bezüglich ihrer Spezifitat, vielfach auf rein physikalische Prozesse (Adsorption an enorm vergrösserte Wirkungsoberflāchen usw.) zurückführen. So ist es leicht verständlich, wenn beispielsweise Autoren wie Loeb gewisse Eiweissverbindungen als Adsorptionsverbindungen auffassen, oder wenn Forscher wie Zangger $u$. a. in den verschiedenen Antitoxinen Kolloidadsorptionsprodukte sehen. - Im Hinblick auf diese Verhältnisse und auf die Bedeutung der Oxydationen für die allgemeine und bakterielle Toxikologie habe ich es unternommen, die Beziehungen der von mir beschriebenen Erythrozytengranula auch zur Immunităt tierexperimentell zu untersuchen. Ich behalte mir vor, über die wichtigen Ergebnisse dieser in den verschiedensten Richtungen ausgeführten Untersuchungen dann eingehender zu berichten, wenn ich ihre Tragweite vollkommen überblicken kann.

\section{III.}

Meine bisherigen Ausführungen haben sich mit der Morphologie und Biologie der Erythrozytengranula und damit der Erythrozyten selbst beschäftigt. Ich glaube nicht, dass die vielseitige Bedeutung der Erythrozytengranula damit anch nur einigermaßen erschöpfend studiert und behandelt ist, zumal mir für meine Untersuchungen nur verhältnismässig einfache und beschränkte Hilfsmittel zur Verfügung standen. Meine Arbeit wăre unvollstăndig, wenn ich die Bedeutung jener Granula nicht auch noch vom Standpunkt der modernen Z ell e n l e h r e aus beleuchten wollte.

Wir haben an dem speziellen Beispiel der Erythrozyten den innigen Zusammenhang zwischen Zellstruktur and Zellfunktion sowie ihre gegenseitige Abhängigkeit kennen gelernt. Diese Erkenntnis, auf die allgemeine Zellenlehre angewendet, sowie das Postulat, die Beziehungen zwischen Struktur und Funktion genügend zu würdigen, haben sich seit $\mathrm{V}$ i r chows epochemachenden Arbeiten mehr und mehr Geltung verschafft. Vor Virchow hat man zu einseitig auf die morphologischen Verhăltnisse der Zelle 
Gewicht gelegt und bald einer Zellmembran, bald dem Protoplasma oder dem Kern die Hauptbedeutung zugeschrieben und vor allem die Struktur dieser Gebilde zu erforschen gesucht. Erst mit der Entwicklung und steten Verbesserung der optischen und färbetechnischen Hilfsmittel gingen diejenigen Beobachtungen und vielseitigeren Forschurigen Hand in Hand, welche die spezielle Bedeutung der einzelnen Zellelemente kennen lehrten und die Wichtigkeit ihrer wechselseitigen Abhängigkeit für die Existenz und die Funktion der Zelle, den zugehörigen Zellverband und damit für den Gesamtorganismús betonten. Die Zelle wurde nun als der Elementarorganismus charakterisiert, als die in sich geschlossene Elementarform, in der sich die hauptsăchlichsten Äusserungen des Lebens: Assimilation, Wachstum, Regeneration und spezifische Funktion abspielten (Virchows Trias der Formatio, Nutritio, Functio). Die Berechtigung, in der Zelle den Elementarorganismus $\mathrm{zu}$ sehen, liegt zum nicht unwesentlichsten Teil in ihrer mehr oder weniger bedingten (sämtliche tierischen und pflanzlichen Zellen) oder in ihrer individuell-unbedingten Autonomie (organoide Protozoen) begründet. Bei den Metazoenzellen z. B. ruht die Bedingtheit ibrer Autonomie zunächst in ihrem Verhältnis zu den gleichartig funktionierenden Zellen ihres Gewebsverbandes, sodann zum Teil in ihı er Abhängigkeit vom Nervensystem, schliesslich auch und bauptsächlich in den für die Gesamtfunktion der Zelle unentbehrlichen Wechselbeziehungen zwischen Kern und Protoplasma, als den wichtigsten Strukturkomplexen der Zelle. Eine individuell-unbedingte Selbständigkeit geht der Metazoenzelle ab. -- Die Bedingtheit der Autonomie bei den Protozoen als organoiden Zellen beruht vornehmlich auf der gegenseitigen Abhängigkeit von Kern und Protoplasma, da nur sie das Leben jeder Zelle mit allen Merkmalen des Lebens gewährleisten können. Ich erinnere hier an die bekannten Versuche von $\mathrm{Nussbaum}$, Verworn u. a., die zeigten, dass die kernhaltigen Anteile zerstückelter Amöben und Infusorien überlebend blieben und sich regenerierten, während die kernlosen Stücke alsbald zugrunde gingen. - Je vielwertiger oder vielseitiger eine Zellfunktion ist, in desto höherem Maße ist sie von Wechselbeziehungen differenter Strukturkomplexe der Zelle abhängig. Vergegenwärtigen wir uns die verschiedenen Haupt- und Nebenfunktionen der Zelle als Ganzes, so werden wir, besonders 
bei Zellen mit verschiedenen spezifischen Funktionen, eine sehr ansehnliche Stufenleiter der verschiedensten Korrelationen zwischen differenten, gegenseitig abhängigen Strukturkomplexen und schliesslich sogar zwischen den verschiedensten Strukturelementen annehmen müssen. So ist das Prinzip der Zellteilung und -vermehrung, wie es der Kern verkörpert, vor allem vom Verhalten der Zentrosomen, Zentralkörperchen, abhängig, deren nicht unwesentliche Selbständigkeit darin zum Ausdruck kommt, dass ihnen die Fahigkeit zur Assimilation und Teilung eigen ist. Bei der Mitose zeigen auch die verschiedenen Chromosomen in ihrer Vermehrungsfähigkeit und bekanntlich in der Wahrung genereller Eigentümlichkeiten relative Selbständigkeit. Vor allem diese, bei der Zellteilung zu beobachtenden Erscheinungen haben u. a. nicht wenig zu dem Zweifel an der Bedeutung der Zelle als Elementarorganismus beigetragen. Die Einführung der Vitalfärbung und die durch ihre zunehmend erhöhte Leistungsfähigkeit vermittelten Erkenntnisse und Schlüsse führten schliesslich zu Auffassungen, wie sie vornehmlich die Altmannsche Granulalehre, sodann Arnolds Plasmosomen-Granulatheorie und die Mitochondrienlehre vertreten. Von der Besprechung der fast unübersehbaren Literatur, die dieses Gebiet betrifft, muss ich absehen, dagegen gebe ich in knappster Zusammenfassung den prinzipiell gleichen Kerninhalt jener drei Theorien wieder. Die Zellen stellen keine Elementarorganismen dar, sondern gewissermaßen Kolonien von kleinsten Elementarorganismen, welche durch Granula verkörpert werden und ihren Ursprung von sukzessive an Grösse zunehmenden gleichartigen Strukturelementen nehmen. Diese Strukturelemente sollen nach Altmann freilebenden Autoblasten (z. B. Mikrokokken) als sogenannte Bioblasten analog sein. Nach A rnold leiten sich jene Granula von Zytoplasmamikrosomen, den sogenannten Plasmosomen, ab und stellen ähnlich den Mitochondrienapparaten (Granulaketten) Bendas u. a. Organellen, Organula dar, denen die Hauptmerkmale des Lebens, wie Assimilation, Wachstum und Regeneration zukommen und an welche gleichzeitig die verschiedenartigsten Stoffwechselvorgänge (Fett-; Eiweiss-, Kohlehydrat- und Pigmentstoffwechsel) gebunden sein können; auch für den Gasstoffwechsel der Zelle sowie für die verschiedenen Oxydationsfermente kommen, wie ich oben angeführt habe, Zellgranula in Betracht. 
Hier ist auf die weitgehenden Analogien $z$ wischen den von mir beschriebenen Erythrozytengranulis und den Chloroplasten der grünen Pflanzen hinzuweisen. Beide vermitteln als Trăger biologisch wichtiger Farbstoffe, des Hämoglobins bezw. des Chlorophylls, die beide im Methylpropylpyrrol àuf eine gemeinsame Mutterverbindung zurückgehen, wichtige chemische Prozesse. Diese chemischen Vorgange sind beim Hämoglobin bekanntlich vorwiegend analytischer, beim Chlorophyll vorzugsweise synthetischer Natur. Für die erstaunliche Massenwirkung des grünen Blattfarbstoffes ist $u$. a. gleichfalls das Prinzip stark vergrösserter Wirkungsoberflächen von grösster Bedeutung (weitgehende Verăstelung, Vergrösserung der Assimilationsoberfläche durch entsprechend zahlreiche, besonders gefiederte oder gekerbte Blătter, Anordnung der Chlorophyllkörner u. a. m.). Es ist von Wichtigkeit, zu bemerken, dass auch die Chloroplasten, ebenso die Leuko- und Chromoplasten, insofern einige Selbständigkeit besitzen, als ihnen die Fähigkeit zukommt, zu assimilieren, zu wachsen und sich durch einfache Zweiteilung innerhalb der Zelle zu vermehren. Ausserdem spielen sich bauptsăchlich auch an ihnen Fermentprozesse $a b$, die für das Leben und den hochorganisierten Betrieb der Pflanzenzelle unentbehrlich sind (z. B. Diastasen und Fermente, welche die Reservestärke in lösliche Glukosen und Maltosen überführen, bezw. diese wiederum in transitorische Stärke usw.).

Alle Gebilde, die man bisher als Altmannsche Granula bezeichnete, ferner die Mitochondrien und ähnlichen granulären Elemente besitzen eine nur relative Selbständigkeit; ihnen allen ist das eine oder andere hervorstechende Merkmal des Lebens und ebenso ihr Ursprung aus einer Mutterzelle eigen. Sie sind auch lediglich als Strukturelemente, funktionell im besten Falle als Organula ihrer Mutterzellen zu betrachten und nur als solche lebensfähig. Ein freies, d. h. extrazelluläres Leben als autonome Individuen, als Autoblasten wie etwa Mikrokokken, ist ihnen schlechterdings unmöglich. Die Wertigkeit der Altmann schen Granula als Bioblasten und als Elementarorganismen der Zelle wurde deshalb bisher von den meisten Forschern mit Recht bestritten. Dagegen weisen meine Untersuchungen den Erythrozytengranulis unter allen bisher bekannten granulären Formelementen der Zelle eine wichtige Sonderstellung zu. Für jene 
Granula sind die Erythrozyten die Bildungs- und Mutterzellen, deren lebenswichtigste Strukturelemente sie bilden. Sie assimilieren, wachsen durch Intuszeption und vermehren sich schliesslich durch Zweiteilung wie die übrigen Granula. Nach meinen Untersuchungen unterscheiden sie sich aber den übrigen Granulis gegenüber ganz prinzipiell dadurch, dass sie auch ausserhalb der Mutterzellen in verhältnismässig anspruchslosen Nährsubstraten als autonome Individuen frei zu leben vermögen. Die Erythrozytengranula sind Autoblasten, den Mikroben analog, welche alle ihre spezifischen Lebensmerkmale ebensogut ausserhalb wie innerhalb ihrer Mutterzellen aussern können, einschliesslich ihrer Făhigkeit, sich durch einfache $Z$ weiteilung zu vermehren und wiederum gleichartige und gleichartig funktionierende, selbständige Individuen $\mathrm{zu}$ bilden. Ihre Biologie stützt Altmanns Hypothese: „Omne granulum e granulo" wenigstens für einen speziellen Fall und damit für Altmanns Granulalehre überhaupt. Meine Untersuchungsergebnisse haben zum erstenmal Beweise geliefert, dass die Zelle Bioblasten im eigentlichen Sinne bergen kann, d. h. kleinere Lebenseinheiten als die Mutterzelle selbst. Die Selbständigkeit der (kultivierten) Erythrozyten granula unterscheidet sich prinzipiell in keiner Weise von derjenigen der autoblastischen Mikrokokken, doch quantitativ insofern, als sich unsere Granula nach der ersten Kultivierung nicht auf weitere Nährböden erfolgreich überimpfen und weiter züchten lassen. Dieser unwesentliche Unterschied ändert aber nichts an der Bedeutung, welche die Biologie der Erythrozytengranula für die Geltung der Altmannschen Granulalehre hat.

Die kultivierten Erythrozytengranula åussern die Hauptfunktionen des Zellprotoplasmas wie Assimilation, Wachstumsreize, spezitische chemische Prozesse (Oxydationsvorgănge), gleichzeitig aber wohnt ihnen auch die Fähigkeit zur Vermehrung durch. Zweiteilung inne, eine "Lebenskraft", die sonst nur durch Vermittlung eines Zellkernes ermöglicht wird. Wir haben also in dem Erythrozytengranulum in funktioneller Hinsicht einen Elementarorganismus vor uns, der wiederum das Homologon, das verkleinerte Abbild seiner Mutterzelle darstellt. Es ist durchaus logisch, die Gleichartigkeit, welche die Erythrozyten. als vollkommene Zellen und ihre isolierten, kultivierten Granula bezüglich ihrer Lebensäusserungen und Funktionen zeigen, in beiden 
Fällen auch auf die Gleichartigkeit von bestimmten, den verschiedenen Lebensäusserungen entsprechenden Strukturanteilen zu beziehen. Sind bei den Mutterzellen, den Erythrozyten, Ernährung, Wachstum und Oxydationsprozesse an das Protoplasma, die Vermehrungsfăhigkeit an den Kern gebunden, so sind dieselben Lebensäusserungen in den freien Erythrozytengranulis an bestimmte Protoplasmaanteile bezw. an bestimmte. Kernanteile fixiert. Die roten Blutzellen der Säugetiere verkörpern demnach tatsächlich in gewissem Sinne Kolonien ron Elementarorganismen verschiedener Ordnungen. Sehr wahrscheinlich ist die physiologische Gesamtwirkung der Mutterzellen gleich den summierten Effekten aller ihrer granularen Elementarorganismen. Dass diese Verhăltnisse, wie wir sie zwischen den Erythrozyten des strömenden Blutes und ihren freien, kultivierten Granulis kennen gelernt haben, vielleicht in komplizierterer Modifikation, auch auf alle übrigen Körperzellen übertragen werden dürfen, dafür lassen sich verschiedene Wahrscheinlichkeitsgründe anführen. Diese Wahrscheinlichkeitsgründe sind in besonderen, engen Bezjehungen zwischen Kern- und Protoplasmasubstanzen gegeben. Nach Boveris Beobachtungen wird bei der Mitose von Eizellen ProtoplasmamateriaI in Kernsubstanz transformiert. Umgekehrt liefern unter Umständen ausgesprochene, mit allen Eigentümlichkeiten der Kernsubstanz ausgestattete Kernderivate "reine“, komplizierte protoplasmatische Strukturen. So entsteht z. B. beim Prozess der Eireifung bei der Rückbildung des Keimbläschens aus gewissen Bestandteilen seines Chromatins eine protoplasmatische Kernspindel. Hierher gehören ferner die Beobachtungen, nach denen kernlose Anteile von Eiern niederer Metazoen (Echinoiden) nach der Befruchtung noch zur Furchung, ja zur Larvenbildung fähig sind. Eine besondere Bedeutung für die Kern- Plasmarelation schreibt R. Hertwig den Chromidien, Goldschmidt dem Chromidialapparat zu, im Protoplasma nachweisbaren, sporadischen oder durch Netze oder Stränge verbundenen chromatinreichen Köornchen. Sehr wahrscheinlich kommen für jene metaplasieähnlichen Vorgänge bei der Eireifung und Zellteilung nicht wirkliche Umwandlungen so differenter Zellanteile, wie sie Kern und Protoplasma vorstellen, in Frage. Sehr vieles spricht nach meinen Untersuchungen dafür, dass die Zelle in morphologischer wie biologischer Beziehung durchgehends nach dem Prinzip der 
Bipolarităt gebaut ist. Darnach besteht jede Zelle nicht nur aus dem streng umgrenzten Kern und dem Protoplasma, sondern es sind in der Masse der ganzen Zelle ausserdem noch mehr oder weniger zahlreiche Komplexe von Kern-und Protoplasmaanteilen diffus verteilt. Wir dürfen annehmen, dass. je nach dem Bedürfnis der Kern- und Plasmarelation bald die Funktionen des protoplasmatischen, bald diejenigen des Kernpoles jener Komplexe prävalieren. Diese Prăvalenz kommt mitunter sehr deutlich in bestimmten Strukturverschiebungen zum Ausdruck und lässt sich dann durch bestimmte Methoden auch färberisch erkennen. Sprechende Beispiele von Zellen, die im Sinne meiner Auffassung bipolar gebaut sind, haben wir in den Säugetiererythrozyten kennen gelernt mit ihren Kern-Protoplasmakomplexen, den Erythrozytengranulis. Die Vererbung allgemeiner Zellmerkmale und -funktionen, die Vererbung im weitesten Sinne, sowie die Regeneration tierischer und besonders auch pflanzlicher Zellen und Gewebe liefern manche Stütze für die Bipolaritătshypothese, welche wiederum diese Vorgănge in neuer und klarerer Beleuchtung erscheinen lässt. - Die bipolare Differenzierung der Zelle und ihrer Organellen und Bioblasten erleichtert es uns ganz wesentlich, auch solchen Gebilden, die keinen streng geformten distinkten Kern aufzuweisen haben wie z. B. gerade die Săugetiererythrozyten, gleichwohl als vollkommene Zellen zu betrachten. Von den eben skizzierten Gesichtspunkten aus făllt auch die Charakterisierung und Rubrizierung der Bakterien von seiten der Zellenlehre nicht mehr schwer. Die Bakterien enthalten statt distinkter Kerne im Protaplasma wechselnd zahlreiche Körnchen, die als Chromatingranula den Chromidien $\mathrm{R}$, Hertwigs gleichwertig sind. Schaudinn setzt sie nach seinen Beobachtungen und Untersuchungen am Bac. Bütschlii einem Kern analog, ebenso nach seinen Beobachtungen am Bac. buccalis maximus $\mathrm{S} w e l l e n g r e b e l$, der für viele Bakterien. ein sogenanntes Amphiplasma mit eingestreuten Chromatinkörnchen annimmt. Schon Vir ch o w hat den Bakterien den Charakter von echten Zellen zugestanden, und wir können auf Grund eingehender Untersuchungen diese Charakterisierung als vollberechtigt anerkennen. Die Annahme ist angebracht, dass auch in der Bakterienzelle Kern- und Protoplasmasubstanzen in morphologischer und funktioneller Hinsicht nach dem Prinzip der 
Bipolarität (bipolare Kern-Plasmakomplexe) angeordnet sind. Die am ehesten verständlichsten Beispiele hierzu liefern besonders die sporenbildenden Bakterien. So stehen z. B. die Tuberkuloseerreger zu ihren sporoiden und sporenäquivalenten Körnern biologisch in einem ähnlichen Verhältnis wie die Säugetiererythrozyten zu ihren kultivierbaren Granulis. Nach unserer heutigen Erkenntnis ist ein Auswachsen von Sporen ohne die entsprechenden Kern- und Protoplasmaanteile zu einer neuen Bakterienzelle mit allen spezifischen Eigenschaften und Merkmalen undenkbar.

Die Hypothese von der Zusammensetzung der Zelle aus bipolaren Kern-Plasmakomplexen ist ein Postulat der Logik, das sich ergibt aus unserer Kenntnis von der Lebenswichtigkeit der Kern- und Protoplasmasubstanzen sowohl für die Mutterzelle als auch für ihre Bioblasten aller Ordnungen, mögen sie ein intrazelluläres oder ein freies extrazelluläres Leben führen. Unsere Hypothese erklärt zwar die Möglichkeit der Entstehung homologer biologischer Einheiten verschiedener Ordnung aus einer Mutterzelle, aber auch sie führt keineswegs zur Kenntnis der histologischen Elementarteile der Zelle. Stehen schon die Trăger der untersten biologischen Lebenseinheiten, wie sie die kleinsten Granula verkörpern, an der Grenze der optischen Wahrnehmbarkeit, wieviel mehr dann erst die - wenn überhaupt darzustellenden - Strukturkomplexe, welche allein die Existenz dieser Lebenseinheiten bedingen! Damit haben wir uns M. Heidenhains Protomerentheorie genähert, welche, die Bedeutung und Berechtigung der oben dargelegten Granulalehre anerkennend, die letzten logischen Konsequenzen zieht. Nach Heidenhain nehmen die Granula als lebende Zellstrukturelemente von den sogenannten Protomeren, den kleinsten und metamikroskopischen Trägern des Lebens ibren Ursprung. Die Masse der lebenden Zelle wie jeder lebenden Substanz überhaupt lässt sich letzten Endes nicht auf histologisch-mikroskopische Elementarstrukturen wie Fibrillen, Waben, Granula zurückführen, sondern auf wirksame Moleküle und Molekularverbindungen, die sich, zunächst an nicht sichtbare Metastrukturen eines homogenen Plasmas gebunden, später zu mikroskopisch differenzierbaren Strukturen entwickeln. Mit dieser Theorie des molekularen Zellenaufbaues lässt sich das Verständnis der vielseitigen, auch der spezifischen Zellfunktionen wohl vereinigen. Wir haben den Beweis dafür, dass 
in den gleichen Granulis gleichzeitig die verschiedenartigsten Reaktionen ablaufen können; es steht nichts im Wege, diese vielseitigen Funktionsfähigkeiten auch für die Protomeren anzunehmen. Voraussetzung sind dann allerdings homologe Moleküle und Molekularverbindungen. Unter Zuhilfenahme verhältnismässig einfacher physikalischer Prinzipien (Diffusion, Osmose, vergrösserte Oberflachen) sowie unter Vermittlung von relativ einfachen Substitutions-, Polymerisations- (Stărke, Glykogen!) und Dissoziationsprozessen gelingt es den Molekularstrukturen des Plasmas, Verbindungen auf- und, abzubauen, die für den Betrieb der Zelle ebenso lebenswichtig sind wie für den Gesamtorganismus überhaupt. Man vergegenwårtige sich hier auch die vielseitigen Analogien zwischen anorganischen und organischen katalytischen Reaktionen, von denen letztere als Enzym- und Fermentprozesse vornehmlich an Granula gebunden sind, man vergegenwärtige sich ferner die vielfach fast spezifische. Giftempfindlichkeit mancher anorganischer und organischer Katalysatoren u. a. m.!

Die gleiche Logik, welche die primitiven Lebensäusserungen der Zelle auf metamikroskopische Protomeren und Mikrosomen als Träger bestimmter Moleküle und Molekularverbindungen eines homogenen Plasmas zurückführt, muss auch gleichzeitig die gebührende Ber ücksichtigung des Formproblems verlangen. Denn alles Leben ist notwendigerweise irgendwie an die Form gebunden, sie bestimmt als wahrer logischer Kern das Wesen des organischen Individuums (F. A. Lange), als einer einheitlichen Gemeinschaft, in der alle Teile zu einem gleichartigen Zweck zusammenwirken oder nach einem bestimmten Plan tätig sind (Virchow). Nur in diesem Sinne dürfen die Zellen und alle Granula, die auch in extrazellulärer Autonomie leben können, gewertet werden, d. h. als wirkliche organische Einheiten und Individuen im Gegensatz zu den bloss gedachten Einheiten wie etwa die Atome, die Protomeren usw. Vielleicht kann meine Arbeit die Erkenntnis festigen, dass auch die Bakterien vollkommene Zellen mit Kern-Plasmakomplexen darstellen. Wir wissen ferner, dass die Zelle die Bildungsstătte von Bioblasten sein kann, durch deren normale Funktion als Organellen allein oder in Verbindung mit anderen Zellfunktionen die Existenz der Mutterzelle gewährleistet wird. Die Existenz der Bildungszelle und ihrer Strukturelemente, mögen sie unselbständige Organellen 
sein oder selbständigere Bioblasten, ist allein bedingt durch ihre wechselseitige Abhängigkeit. Dieses alles lässt uns einséhen, dass alle Physiologie im letzten Grunde Zellularphysiologie sein muss, ebenso wie alle Pathologie nach der genial umfassenden Erkenntnis Virchows im wesentlichen immer Z ellularpatbologi e bleiben wird.

\section{Zusammenfassung.}

1. Die Erythrozyten der Säugetiere sind vollk om mene Z ellen, bestehend aus Protoplasma und einem echten Nukleoid, dem zentralen Chromatinkörper, der dem färberisch direkt nachweisbaren Kern anderer Zellen âquivalent ist.

2. Wichtige Strukturelemente der Saugetiererythrozyten stellen Granula dar, die sich mit Hilfe der einfachen oder kombinierten Gramfärbung nachweisen lassen. Bei den verschiedenen funktionellen Schădidungen des Blutes fehlt das normale granuläre Strukturbild der roten Blutzellen entweder überhaupt, oder es zeigt ausgesprochene Veränderungen.

3. Es gelingt, die.Granula explantierter Erythrozyten in bestimmten Nährmedien zu kultivieren, zur Vermehrung durch Zweiteilung und zu intensivem Massenwachstum zu veranlassen.

4. Durchintravenöse Injektionkultivierter Erythrozytengranula wird die Gerinnungsfähigkeit des Versuchstierblutes deutlich herabgesetzt oder völlig a u f e hoben.

5. Die Granula der roten Blutzellen sind kolloidaler Natur und hämoglobinhaltig; sie wirken katalytisch und sind die Träger kräftiger Oxydationsprozesse; vor allem sind nachweisbar Oxydasen, vermutlich auch Katalasen an sie gebunden.

6. Die erstaunlichen Wirkungen der Oxydationsprozesse im Säugetierorganismus sind zu einem sehr wesentlichen Teil zurückzuführen auf das Prinzip enorm vergrösserter Wirkungsoberflächen, wie es beim Sauerstoff spendenden Zirkulationsapparat in gleicher Weise wie beim Sauerstoff empfangenden 
System der lebenswichtigen Gewebe und Organe bis an die ausserste Grenze der Möglichkeit ausgebildet ist. Die granulăre Struktur der Säugetiererythrozyten unterstützt das Prinzip der Oberflăchenvergrösserung in weitgehendstem $\mathrm{M}$ a B e.

7. Auf die katalytische Wirkung der Erythrozytengranula sind die günstigen. Erfolge der Bluttransfusion zu beziehen bei der akuten, posthămorrhagischen Anämie und bei der Kohlenoxydvergiftung.

8. Die Beeintrăchtigung der Gerinnungsfähig$k$ i t des Versuchstierblutes nach Injektion kultivierter Erythrozytengranula kommt wahrscheinlich d u r ch fermentative Fettspaltungen im Blutplasma zustande. Auch beim Fieber, bei der Blutgerinnung und bei gewissen Immunprozessen spielen erhöhte Oxydationsvorgänge mit konsekutiven. Fermentwirkungen eine unverkennbare Rolle.

9. In den Erythrozytengranulis sehen wir zum ersten Mal granuläre Strukturelemente einer Zelle, welche die hauptsächlichsten Lebensfunktionen (Assimilation, Wachstum, Vermehrung) nicht nur als intrazelluläre Organula äussern, sondern auch die Fähigkeit besitzen, diese Lebensfunktionen in extrazellularer A utonomie geltend zu machen.

10. Die Erythrozytengranula dürfen als Bioblasten bezeichnet werden, deren Biologie eine Hauptstütze für die Altmannsche Granulalehremit allen ihren Folgerungen liefert.

\section{Literaturverzeichnis.}

A b derbalden: Bau und Stoffwechsel der Zelle, Berlin 1911; Physiol. Chemie 1906.

A Itman n: Elementarorganismen, Studien über die Zelle, Leipzig 1886, 1890, 1894.

Arloing und La alasnie: La Sem. méd. 1895.

Arnold: Virchows Archiv 145, 153, 166 ff.; Zentralblatt f. Path. 1908.

Batelli und Stern, Compt. rend. Soc. Biol. 58. 
Benda: Physiolog. Gesellschaft Berlin 1899, 1900; Anat. Gesellsch. Heidelberg 1903.

Bordet und Delange: Arch. f. exp. Path. u. Pharm. 71.

Bremer: Arch. f. mikrosk. Anat. 45.

Büt $\mathrm{schli}$, Mikroskop. Schäume u. d. Plasma 1892.

Do y on a. Dufourt: Arch. d. physiol. 30 .

Ferrata, Fol. haemat. Arch. 9.

Gierke, M. m. W. 1911.

Goldmann: Beitr. z. klin. Chirurgie 64, 78; Vitalfärbung d. Zentralnervensystems, Akad. d. Wissensch. Berlin 1913.

Goids chmidt: Biol. Zentralblatt 21, 24 ; Arch. f. Protistenkunde 5.

Gross o: Fol. haemat. Arch. 18.

Heide $\mathrm{nh}$ a in: Plasma und Zelle, Jena 1907, 1911.

Hertwig: Sitzungsber. d. morphol. u. physiol. Gesellschaft, München 1903; Biol. Zentralblatt 1902.

Horbaczewski: Monatsh. f. Chemie 10, 12.

Kronberger: Fol. haemat. Arch. 13.

Lange: Geschichte des Materialismus, Stuttgart 1907.

Lavdowsky: Zeitschr. f. wiss. Mikr. 10.

L u barsch: Eulenburgs Realenzykl. 1908.

Murath: M. m. W. 1917.

Me ves, Arch: f. mikrosk. Anat. $48 \mathrm{ff}$.

$\mathrm{N}$ ägeli: Fol. haemat. 5 .

N u s s baum: Areh. f. mikrosk. Anat. 26.

Orth: Virchows Arch. 200.

Pappen heim: Folia clin. 1.

Ribbert: Zeitschr. f. allg. Physiol. 4.

Russel: Brit. Med. Journ. 1890.

Schilling-Torga a: Fol. haematt. 11; M. m. W. 1911.

Schmidt, D. p. G. 15, Strasshurg 1912.

Schultze, Arch. f. mikrosk. Anat. 1.

Schridde: Arch. f. Derm. u. Syph. 73; Zentralbl. f. Path. 76.

Spitzer: Pflügers Arch. 67.

Stuber: Biochem. Zeitschr. 77.

Tü rk: Vorlesungen über klin. Hämatol. 1912.

Verworn: Pflügers Archiv 48.

Vir chow: Zellularpathologie 1858; Virchows Archiv 4, 150 ff.

W a rbu rg, Ergebn. d. Physiol. 14.

Wederhake, M. m. W. 1917.

Zack: Arch. f. exp. Path. n. Pharm. 74.

Z angger, Vierteljahrschr. d. naturf. Gesellsch. Zürich 1908; Zeitschr. f. Immunitätsforschung 1 . 